\title{
Comparing visual search and eye movements in bilinguals and monolinguals
}

\author{
Ileana Ratiu ${ }^{1,2}$ • Michael C. Hout ${ }^{3}$ - Stephen C. Walenchok ${ }^{1} \cdot$ Tamiko Azuma $^{1}$. \\ Stephen D. Goldinger ${ }^{1}$
}

Published online: 15 May 2017

(C) The Psychonomic Society, Inc. 2017

\begin{abstract}
Recent research has suggested that bilinguals show advantages over monolinguals in visual search tasks, although these findings have been derived from global behavioral measures of accuracy and response times. In the present study we sought to explore the bilingual advantage by using more sensitive eyetracking techniques across three visual search experiments. These spatially and temporally fine-grained measures allowed us to carefully investigate any nuanced attentional differences between bilinguals and monolinguals. Bilingual and monolingual participants completed visual search tasks that varied in difficulty. The experiments required participants to make careful discriminations in order to detect target Landolt Cs among similar distractors. In Experiment 1, participants performed both feature and conjunction search. In Experiments 2 and 3, participants performed visual search while making different types of speeded discriminations, after either locating the target or mentally updating a constantly changing target. The results across all experiments revealed that bilinguals and monolinguals were equally efficient at guiding attention and generating responses. These findings suggest that the bilingual advantage does not reflect a general benefit in attentional guidance, but could reflect more efficient guidance only under specific task demands.
\end{abstract}

Keywords Visual search $\cdot$ Eye movements $\cdot$ Attentional control $\cdot$ Bilingualism

Ileana Ratiu

iratiu@midwestern.edu

Arizona State University, Tempe, AZ, USA

2 Midwestern University, Glendale, AZ, USA

3 New Mexico State University, Las Cruces, NM, USA
Bilingual individuals regularly manage two or more languages efficiently. They are able to converse in one language while inhibiting the other(s), and even switch languages within a conversation with minimal error (Gollan, Sandoval, \& Salmon, 2011). Thus, for bilingual individuals, language selection is theorized to be a near-constant demand on executive resources. Consistent with this view, many studies have suggested that lifelong bilingualism fosters general cognitive advantages. Previous studies have reported a bilingual advantage in nonlinguistic tasks that require the inhibition of irrelevant stimuli (Bialystok, Craik, \& Ryan, 2006; Emmorey, Luk, Pyers, \& Bialystok, 2008), updating and monitoring of information (Bialystok, Craik, \& Ruocco, 2006; Costa, Hernández, Costa-Faidella, \& Sebastián-Gallés, 2009), and shifting or switching between tasks (Prior \& Gollan, 2011; Prior \& MacWhinney, 2010).

Previous research has demonstrated that, for bilinguals, lexical items in both languages are always active, regardless of the language context (e.g., De Groot, Delmaar, \& Lupker, 2000; Dijkstra, Van Jaarsveld, \& Ten Brinke, 1998; Kroll, Bobb, Misra, \& Guo, 2008). For instance, when a SpanishEnglish bilingual thinks about the concept tree, representations for both "tree" and "árbol" will become active. The speaker cannot simultaneously say "tree" and "árbol," so these items compete for lexical selection. Green and Abutalebi (2013) proposed that bilinguals use multiple adaptive control mechanisms to resolve the conflict between competing language activations, referred to as language control. According to their adaptive-control hypothesis, bilinguals use processes such as goal maintenance, interference suppression, response inhibition, and task engagement/disengagement to manage their languages in different linguistic contexts. These mechanisms are associated with one or more executive functions. Although the adaptive-control hypothesis primarily makes assumptions about language processing, Green and 
Abutalebi suggested that a cognitive advantage may arise for bilinguals because language control requires general cognitive mechanisms that become more efficient under the constant demands of bilingual life. By virtue of exercising constant language selection, bilinguals may develop general cognitive or executive function advantages, even in nonlinguistic domains (e.g., Bialystok, Craik, \& Ryan, 2006).

Executive functions (EFs) denote a set of cognitive processes responsible for the complex control of thoughts and actions. People require EFs when suppressing interference from distracting stimuli, holding goals in memory, or switching attention between tasks. Individual differences in EF are well-documented, and these differences often predict performance in cognitive tasks (Friedman et al., 2006). Closely related to EF is working memory capacity (WMC; see, e.g., Miyake et al., 2000), which allows individuals to attend to goal-relevant information (Shipstead, Lindsey, Marshall, \& Engle, 2014). Although there is ongoing debate regarding the exact relationship between WMC and EF, higher WMC is associated with better performance across many cognitive tasks (e.g., Kane \& Engle, 2002). WMC may be affected by second language acquisition. Macnamara and Conway (2014) found that some aspects of WMC improved with second language acquisition and concluded that bilinguals with high language control demands (i.e., frequent use of both languages) have enhanced cognitive control and WMC.

The benefits of higher WMC and bilingualism may also extend to visual search tasks (e.g., Friesen, Latman, Calvo, \& Bialystok, 2015; Hernández, Costa, \& Humphreys, 2012; Poole \& Kane, 2009; Sobel, Gerrie, Poole, \& Kane, 2007). Visual search is a flexible paradigm that has the potential to help address theoretical questions regarding EF. Searching requires people to judiciously guide attention to the right locations while simultaneously ignoring distracting information. It also requires that people maintain task goals in memory, and match mental templates to visual input. Search difficulty can be manipulated by varying the number of items in the display, changing target discrimination difficulty among the distractors, or imposing simultaneous tasks. Previous research has shown that people with high WMC located targets faster than people with low WMC when the targets were embedded among highly similar distractors (e.g., Sobel et al., 2007), and while holding multiple potential target locations in working memory for long durations (e.g., Poole \& Kane, 2009). However, when targets were highly discriminable from distractors, no WMC advantage was observed (e.g., Kane, Poole, Tuholski, \& Engle, 2006).

Relatedly, there is evidence that bilinguals may have an advantage over monolinguals in visual search tasks. Hernández et al. (2012) found that young adult SpanishCatalan bilinguals deployed top-down attentional guidance more efficiently than Spanish monolinguals in visual search. Prior to each search trial, their participants were shown a tilted line embedded in a shape and were then required to locate that same line in an array. Critically, the tilted line was located either in the cued shape (a benefit) or a different shape (a cost). Overall, bilinguals responded faster than monolinguals and showed no effect of cueing. The authors suggested that bilinguals rely more on top-down attentional guidance, and can better avoid distraction from irrelevant items.

Friesen and colleagues (2015) compared young adult bilinguals and monolinguals in feature and conjunction search tasks. In feature search, target shapes differed from distractors by only one feature (e.g., color). In conjunction search, targets differed by two features (e.g., color and shape; Treisman \& Gelade, 1980). The authors also manipulated discriminability by making distractors more or less similar to targets in color. A bilingual advantage was only observed in the most difficult condition (low discriminability, conjunction search); the authors suggested that bilinguals are more efficient at engaging and disengaging attention to visual objects.

Chabal, Schroeder, and Marian (2015) examined visual search performance between bilinguals and monolinguals using an audiovisual task. Participants heard the name of a target then proceeded to look for that item (e.g., hearing "dog" and searching for a picture of a dog). During search, they heard sounds that were consistent with the target (e.g., barking), consistent with a distractor in the display, unrelated to any item in the display, or they heard no sound. Their findings showed that bilinguals fixated targets faster than monolinguals, suggesting they had more efficient attentional guidance. Additionally, visual search response times were correlated with performance on EF tasks for the bilingual group, but not for the monolingual group. The authors concluded that bilinguals use EF to facilitate relevant information and downregulate distracting or irrelevant information.

Behavioral measures of visual search have indicated advantages for bilinguals, but the relative coarseness of reaction time and accuracy measures leaves open the possibility that more nuanced conclusions may be drawn by using more fine grained eyetracking measures. In particular, eyetracking may be useful in examining potentially subtle differences between bilingual and monolingual young adults. Although behavioral measures have suggested a bilingual advantage in children and older adults (e.g., Bialystok \& Viswanathan, 2009; Emmorey et al., 2008), the results of studies on young adults have been less consistent (e.g., Hilchey \& Klein, 2011; Paap \& Greenberg, 2013; Paap, Johnson, \& Sawi, 2015; Ratiu \& Azuma, 2015). For example, Paap and Greenberg examined whether a bilingual advantage exists in inhibition, monitoring, and task switching abilities using multiple executive function tasks, including an antisaccade task, Simon task, and color-shape switch task. However, the authors found no evidence of a bilingual advantage in any of these tasks. Paap and Greenberg argued that the executive function advantage attributed to bilingualism could be explained by other factors, such as task-specific performance, or demographic factors 
(including age). Similarly, Ratiu and Azuma examined the bilingual advantage in WMC, but did not observe a bilingual advantage in any task. In a recent review, Paap et al. (2015) argued that the bilingual advantage could reflect publication bias, differences in socioeconomic status, immigrant status, questionable statistics, and tasks that are not sensitive to individual differences in domain-general processes.

These previous investigations have used the behavioral measures of accuracy and response time, which may lack the sensitivity required to detect a bilingual advantage in young participants who are at their cognitive peak (Bialystok, 2006; Costa et al., 2009; Kousaie \& Phillips, 2012; Paap \& Greenberg, 2013). Eyetracking measurements permit more sensitive, realtime examination of cognitive processing during visual search and can allow us to explore the purported bilingual advantage more closely.

Prior studies using eyetracking to examine bilingual cognitive advantages have produced mixed findings. In an antisaccade task, Bialystok, Craik, and Ryan (2006) found that bilinguals responded faster than monolinguals when the task included a keypress, but the groups did not differ in their eye movements. The authors proposed that bilinguals had more efficient response initiation. In a related study, Singh and Mishra (2012, 2013) compared low- and high-proficiency Hindi-English bilinguals, measuring saccade latencies in an oculomotor version of the Stroop task. Participants were required to fixate on colored squares that were consistent with cued colors but inconsistent with cued directions (e.g., a downward red arrow, paired with a target red square located above the cue). More proficient bilinguals were faster at initiating correct saccades, suggesting that bilingual proficiency may affect attention control.

In visual search, successful performance requires a person to efficiently guide attention to potential target objects and then make swift, accurate decisions about each inspected item (e.g., Godwin, Walenchok, Houpt, Hout, \& Goldinger, 2015). Previous findings suggested that bilinguals are more efficient than monolinguals at finding target stimuli embedded among distractors (Friesen et al., 2015; Hernández et al., 2012). This visual search advantage may reflect better attentional guidance or faster response initiation (as was suggested by Hilchey \& Klein, 2011), or a combination of both. The specific process(es) underlying the bilingual advantage shown in prior visual search studies cannot be determined, as overall response times (RTs) share contributions from both processes (see Hout \& Goldinger, 2015; Hout, Walenchok, Goldinger, \& Wolfe, 2015). Friesen et al. (2015) recommended that "Future research should employ eye-tracking techniques to investigate whether it is the time course of engaging and disengaging attention when scanning the display that is more rapid for bilinguals or whether bilinguals are better able to initiate a response once the target has been found" (p. 700).

The present experiments were designed to explore visual search differences between monolinguals and bilinguals using behavioral data and participants' eye movements. Each task required participants to make careful discriminations to detect target items among similar distractors. In Experiment 1, participants searched for a target embedded among distractors that were dissimilar (feature search) or similar (conjunction search). In Experiments 2 and 3, participants performed visual search while making speeded discriminations after locating the target, and mentally updating a constantly changing target.

\section{Experiment 1}

In Experiment 1, we examined bilingual and monolingual performance on feature and conjunction visual search tasks. Previous studies have reported that bilinguals outperform monolinguals on visual search tasks that require the inhibition of irrelevant or distracting stimuli (e.g., Costa et al., 2009; Emmorey et al., 2008). In this experiment, participants searched for a Landolt $\mathrm{C}$ target embedded among Os and Landolt Cs of different orientations. Search difficulty was manipulated by varying the similarity of distractors to the target, both in shape and in color shades. Previous studies documenting a bilingual search advantage used color stimuli (e.g., Friesen et al., 2015; Hernández et al., 2012). Thus, it is possible that bilinguals were more efficient at locating targets because they were better at using colors to guide search. To address this possibility, one condition in our experiment contained all black stimuli. Additionally, participants completed working memory and fluid intelligence tasks (symmetry span task and Raven's Advanced Progressive Matrices, respectively) to ensure that any possible differences in visual search performance would not be attributable to other cognitive differences.

It was expected that performance would decline for all participants with increased search difficulty (i.e., higher similarity among distractors and targets). If a bilingual search advantage arises from more efficient attentional guidance, bilinguals should show faster first fixation times to targets, relative to monolinguals. If a bilingual advantage arises from more efficient response initiation, bilinguals should have faster decision times (latency from the first fixation to the keypress) than monolinguals. If the advantage arises from a combination of these processes, then bilinguals should show faster overall search RTs (latency from trial onset to the keypress). Given that the participants were young adults, any bilingual differences should be most evident in the difficult conjunction trials (e.g., Friesen et al., 2015).

\section{Method}

\section{Participants}

All participants were recruited from Arizona State University undergraduate classes and received partial course credit for 
participation. Participants were recruited using a general statement regarding the description of the study and criteria for participation. Separate recruitment materials were created for monolinguals and bilinguals, and participants were not provided information regarding the hypotheses until debriefing. Bilingual participants reported speaking English and one other language fluently and reported no history of memory, language, or neurological problems. The Ishihara color vision test was used to determine color blindness (Ishihara, 1917). A modified version of the Language Experience and Proficiency Questionnaire (LEAP-Q; Marian, Blumenfeld, \& Kaushanskaya, 2007) was used to measure relative language fluency for the bilingual participants (see Table 1). All participants gave informed consent, and all procedures were approved by the Arizona State University Human Subjects Institutional Review Board.

Experiment 1 included 15 English monolingual speakers and 15 bilingual participants. The bilinguals self-reported speaking English and one of the following languages: Mandarin (6), Korean (3), other (6). The monolingual group had four females, a mean age of 19.7 years $(S D=2.0)$, and mean education of 13.5 years $(S D=0.74)$. The bilingual group featured five females, a mean age of 20.0 years $(S D=2.4)$, and mean education of 13.6 years $(S D=0.90)$. The groups did not significantly differ in age, education, or maternal education (all $t \mathrm{~s}<1$ ). ${ }^{1}$

\section{Stimuli}

In both the black and the color conditions, the target was always a Landolt $\mathrm{C}$ with a gap on the top, bottom, left, or right. The three levels of trial difficulty (easy, medium, difficult) differed in the degrees of similarity among the targets and distractors (e.g., Duncan \& Humphreys, 1989).

Black condition All stimuli were black. The distractors consisted of circles and/or Landolt Cs with gaps at different orientations than the target. In easy trials, the distractors consisted of $100 \%$ circles. In medium trials, the distractors were primarily circles with four Cs in each search display (one per quadrant). In target-present trials, one of the Cs was the target. In difficult trials, the distractors were all Cs that mismatched the target orientation.

Color condition The distractors were Landolt Cs that mismatched the target in gap orientation, color, or both. Colors were selected from 16 possible colors that composed a "wheel" of hues, wherein each color was equally different from its neighbors in color space (Stroud, Menneer, Cave, \&

\footnotetext{
${ }^{1}$ Maternal education ranged from 1 to $8(1=$ junior high $/$ middle school, $2=$ some high school, $3=$ high school diploma, $4=$ some college, $5=$ associate's degree, $6=$ bachelor's degree, $7=$ master's degree, $8=$ doctorate $/$ professional degree).
}

Donnelly, 2012; see Fig. 7 in Appendix H). Target colors were randomly selected from four possible (equidistant) locations on the wheel, roughly corresponding to "orange," "green," "blue," and "pink." In the easy condition, no distractors matched the target color, making it similar to a "pop-out" search task (Treisman \& Gelade, 1980). In the medium condition, distractors were equally sampled from all four target colors and all four orientations. In the difficult condition, distractor colors were drawn from the same categories as targets (e.g., "oranges"). One-third of the distractors exactly matched the target color, and the remaining distractors differed by only one step in color space. Distractor orientations were randomly sampled with the constraint that the targetcolored distractors had a different orientation than the target.

\section{Procedure}

All visual search experiments were presented stimuli using EPrime 2.0 software (Schneider, Eschman, \& Zuccolotto, 2012). Data were collected using a Dell Optiplex 755 PC. The display was a 21 -in. NEC FE2111SB CRT monitor (20 in. viewable), with resolution set to $1,280 \times 1,024$ and a $75-\mathrm{Hz}$ refresh rate. Eye movements were recorded using a desktop-mounted EyeLink 1000 eyetracker (SR Research Ltd., Mississauga, Ontario, Canada). Temporal resolution was $500 \mathrm{~Hz}$, and spatial resolution was $0.01^{\circ}$. Participants were initially calibrated to ensure accurate tracking and used a chin rest during all search trials. Periodic drift correction and recalibrations were used to ensure accurate recording of gaze position. The Landolt $\mathrm{C}$ stimuli subtended approximately $1^{\circ} \times 1^{\circ}$ (centered).

Symmetry span task (Unsworth, Heitz, Schrock, \& Engle, 2005) Participants were shown black-and-white images and reported whether each was symmetrical around its vertical axis. To indicate their response, participants clicked on the image with a computer mouse, which triggered YES and NO options to appear. Images appeared for $4,000 \mathrm{~ms}$ or until the mouse click. After entering a response, participants saw a $4 \times 4$ matrix with one square shaded red for 1,000 ms. Following the matrix, another image appeared for symmetry judgment. At a recall prompt, participants indicated the order and location of the red squares by clicking on squares in a blank grid, then received accuracy feedback. Sets contained two to six symmetry-matrix pairs with two trials per span length (i.e., number of correctly recalled shaded squares), presented in random order.

Raven's Advanced Progressive Matrices All participants completed an abbreviated version of Raven's Advanced Progressive Matrices (RAPM; Raven, Raven, \& Court, 1998), a measure of general fluid intelligence. In each trial, participants saw displays of $3 \times 3$ matrices of geometric patterns with the bottom right pattern missing. Participants selected a pattern to complete the series from a set of eight possibilities. 
Table 1 Language profile for bilingual participants in all experiments

\begin{tabular}{|c|c|c|c|}
\hline \multicolumn{2}{|c|}{ Self-Ratings for Language 1} & \multicolumn{2}{|c|}{ Self-Ratings for Language 2} \\
\hline \multicolumn{4}{|l|}{ Experiment 1} \\
\hline Daily use & $64.33 \%(15.91)$ & Daily use & $32.67 \%(15.91)$ \\
\hline Daily reading & $71.43 \%(29.92)$ & Daily reading & $25.71 \%(24.14)$ \\
\hline Age of acquisition & $0.33(0.90)$ & Age of acquisition & $7.40(4.39)$ \\
\hline Age fluent & $6.07(3.65)$ & Age fluent & $12.43(6.24)$ \\
\hline Proficiency ${ }^{\mathrm{a}}$ & & Proficiency $^{\mathrm{a}}$ & \\
\hline Speaking & $9.80(0.41)$ & Speaking & $7.00(1.57)$ \\
\hline Understanding & $9.73(0.46)$ & Understanding & $7.66(1.80)$ \\
\hline Reading & $9.60(0.63)$ & Reading & $5.93(2.40)$ \\
\hline \multicolumn{4}{|l|}{ Experiment 2} \\
\hline Daily use & $83.33 \%(12.80)$ & Daily use & $15.83 \%(13.05)$ \\
\hline Daily reading & $89.17 \%(13.96)$ & Daily reading & $10.56 \%(13.81)$ \\
\hline Age of acquisition & $0.56(1.62)$ & Age of acquisition & $7.11(4.19)$ \\
\hline Age fluent & $3.28(3.37)$ & Age fluent & $10.31(4.44)$ \\
\hline Proficiency $^{\mathrm{a}}$ & & Proficiency $^{\mathrm{a}}$ & \\
\hline Speaking & $9.44(0.78)$ & Speaking & $7.89(1.45)$ \\
\hline Understanding & $9.72(0.46)$ & Understanding & $8.56(1.33)$ \\
\hline Reading & $9.61(0.70)$ & Reading & $7.56(1.92)$ \\
\hline \multicolumn{4}{|l|}{ Experiment 3} \\
\hline Daily use & $71.37 \%(17.12)$ & Daily use & $28.62 \%(17.12)$ \\
\hline Daily reading & $76.93 \%(24.40)$ & Daily reading & $23.07 \%(24.34)$ \\
\hline Age of acquisition & $1.24(3.12)$ & Age of acquisition & $6.59(5.48)$ \\
\hline Age fluent & $5.41(4.66)$ & Age fluent & $10.53(6.03)$ \\
\hline Proficiency ${ }^{\mathrm{a}}$ & & Proficiency $^{\mathrm{a}}$ & \\
\hline Speaking & $9.53(0.65)$ & Speaking & $7.19(1.96)$ \\
\hline Understanding & $9.83(0.47)$ & Understanding & $8.10(2.23)$ \\
\hline Reading & $9.62(0.72)$ & Reading & $7.00(2.51)$ \\
\hline
\end{tabular}

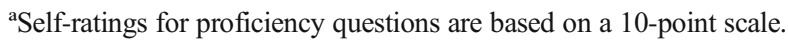

Note: Standard deviations are in parentheses.
The task continued until 18 problems were completed (the trials progressively increased in difficulty) or $10 \mathrm{~min}$ had elapsed.

Visual search After completing 12 practice trials, each participant completed 54 black trials, followed by three blocks of 36 color trials. In all conditions, each trial contained an initial cue screen showing the target Landolt $\mathrm{C}$ (randomly selected in each trial); the cue remained visible until the participant pressed the space bar to begin the search. A central fixation cross was displayed until the participant had looked directly at it for 500 $\mathrm{ms}$, after which the search display appeared. The display contained 16, 20, or 24 images. Targets appeared in half of the trials in random locations. Participants were instructed to press the space bar when they had located the target or decided that the target was not present, with a maximum of $15 \mathrm{~s}$ to complete each trial. After they had pressed the space bar, the search display disappeared. Participants indicated target presence or absence by pressing the " $\mathrm{f}$ " or " $\mathrm{j}$ " keys, respectively, on a standard keyboard. Feedback was given after each trial (see Fig. 1 for the progression of events per trial). After completing the visual search experiment, participants completed the symmetry span and RAPM tasks, in counterbalanced orders.

\section{Results}

In all experiments, standard visual search effects were observed. RTs were slower in the target-absent than in the target-present trials (Exp. 1), and RTs were slower when the distractors were more similar to the target (Exps. 1-3). This confirms that our search difficulty manipulations were effective. For brevity, we 


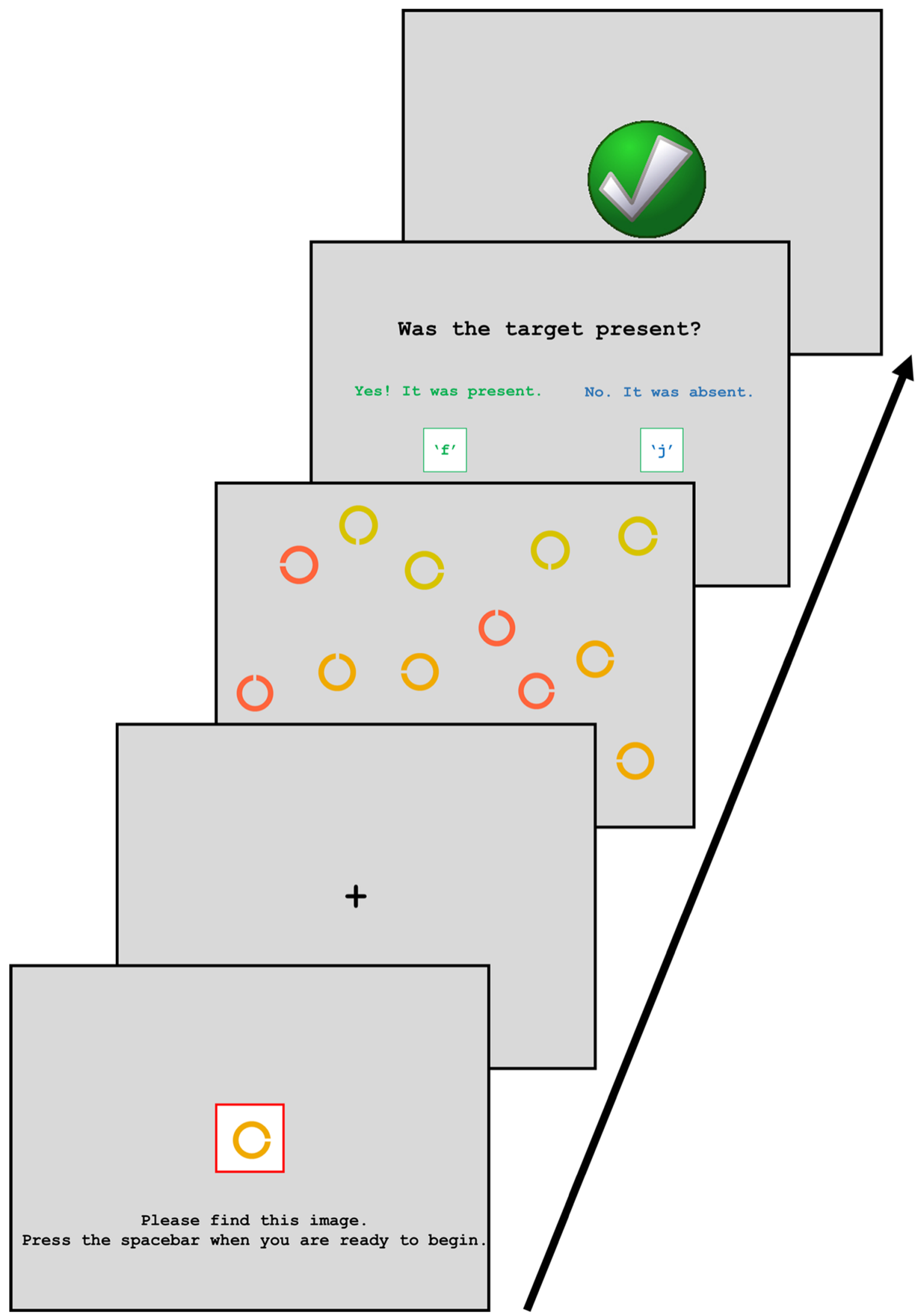

Fig. 1 Progression of trial events in Experiment 1 
Table 2 Mean search times (in milliseconds) for target-present trials in Experiment 1

\begin{tabular}{|c|c|c|c|c|c|c|}
\hline & \multicolumn{3}{|l|}{ Black } & \multicolumn{3}{|l|}{ Color } \\
\hline & Easy & Medium & Difficult & Easy & Medium & Difficult \\
\hline \multicolumn{7}{|l|}{ Overall Search } \\
\hline \multirow[t]{2}{*}{ Bilingual } & $1,715.50(422.25)$ & $2,090.12(668.14)$ & $2,881.06(750.37)$ & 995.37 (210.98) & $1,797.07(393.54)$ & $2,512.67(637.29)$ \\
\hline & {$[1,466.34,1,964.66]$} & {$[1,751.20,2,429.03]$} & {$[2,443.07,3,319.05]$} & {$[875.30,1,115.44]$} & {$[1,608.08,1,986.05]$} & {$[2,192.19,2,833.16]$} \\
\hline \multirow[t]{2}{*}{ Monolingual } & $1,790.30(515.32)$ & $2,138.51(612.24)$ & 3,281.11 (899.18) & 967.17 (241.99) & $1,750.01(316.98)$ & $2,517.84(572.91)$ \\
\hline & {$[1,541.14,2,039.45]$} & {$[1,799.60,2,477.43]$} & {$[2,843.11,3,719.10]$} & {$[847.11,1,087.24]$} & {$[1,561.03,1,938.99]$} & {$[2,197.35,2,838.33]$} \\
\hline \multicolumn{7}{|l|}{ First Fixations } \\
\hline \multirow[t]{2}{*}{ Bilingual } & $1,105.91(333.46)$ & $1,478.58(422.30)$ & $2,360.47(658.48)$ & $507.71(106.96)$ & $1,077.66(247.02)$ & $1,773.77(478.94)$ \\
\hline & {$[932.12,1,279.69]$} & {$[1,267.88,1,689.27]$} & {$[2,012.33,2,708.62]$} & {$[453.78,561.65]$} & {$[963.96,1,191.35]$} & {$[1,561.47,1,986.07]$} \\
\hline \multirow[t]{2}{*}{ Monolingual } & $1,225.73(323.62)$ & $1,469.54(372.91)$ & $2,307.18(658.01)$ & 466.07 (96.74) & $1,107.51(177.21)$ & $1,630.73(304.70)$ \\
\hline & {$[1,051.95,1,399.51]$} & {$[1,258.85,1,680.24]$} & {$[1,959.04,2,655.32]$} & {$[412.14,520.01]$} & {$[993.81,1,221.20]$} & {$[1,418.43,1,843.02]$} \\
\hline \multicolumn{7}{|l|}{ Decision Times } \\
\hline \multirow[t]{2}{*}{ Bilingual } & $687.94(334.46)$ & $758.28(422.88)$ & $795.95(352.22)$ & $506.65(174.65)$ & 737.15 (254.99) & $909.09(294.91)$ \\
\hline & {$[514.57,861.30]$} & {$[552.35,964.20]$} & {$[546.01,1,045.89]$} & {$[408.89,604.43]$} & {$[608.59,865.71]$} & {$[699.90,1,118.29]$} \\
\hline \multirow[t]{2}{*}{ Monolingual } & 670.24 (320.97) & $735.03(352.34)$ & 1,037.44 (567.96) & $510.45(181.59)$ & $689.88(213.60)$ & $1,017.40(447.10)$ \\
\hline & {$[496.87,843.60]$} & {$[529.10,940.95]$} & {$[787.50,1,287.38]$} & {$[415.99,604.90]$} & {$[565.68,814.08]$} & {$[815.30,1,219.50]$} \\
\hline
\end{tabular}

Standard deviations are in parentheses; 95\% confidence intervals are shown in brackets.

focus our reporting on the main effects and interactions involving speaker group, since these were the analyses of interest. The full statistics for RTs are reported in Table 2 and Appendix Table 3. The same analyses were conducted on accuracy. For accuracy, no significant main effects of speaker group emerged for any measure (Exps. 1-3), nor reliable interactions between speaker group and the other factors. Additionally, performance was at $94 \%$ or higher in every experiment, raising concerns about ceiling effects and making the analyses difficult to interpret. Thus, we do not report the accuracy data.

\section{Normative measures}

There was no difference between bilinguals and monolinguals on $\operatorname{RAPM}[t(29)=1.13, p=.269]$. Bilinguals performed marginally better than monolinguals on the symmetry span task $[t(29)=$ $1.89, p=.068]$.

\section{Visual search}

Overall search RTs, first fixation times, and decision times are shown in Table 2. A 2 (Trial Type: absent or present) $\times 3$ (Search Difficulty: easy, medium, or difficult) $\times 2$ (Speaker Group: monolingual or bilingual) mixed-model analysis of variance (ANOVA) was conducted on the mean correct RTs. For first fixation and decision times, a 3 (Search Difficulty: easy, medium, or difficult) $\times 2$ (Speaker Group: monolingual or bilingual) mixed-model ANOVA was conducted on targetpresent trials only (see Appendix Table 3 for the main effects and interactions). All post-hoc pairwise comparisons were evaluated using a Bonferroni-corrected alpha of .017 for multiple comparisons. All ANOVA results, including the effect sizes, are listed in Appendix Table 3. Individual RTs across all conditions (Exps. 1-3) are shown in Appendix I, Figs. 8, 9, 10, 11, 12, 13, 14, 15, 16, 17, 18 and 19.

\section{Overall search $R T$}

The main effect of speaker group was not reliable in either color condition. No other interactions were reliable. ${ }^{2}$

\section{First fixation times}

In both color conditions, only the main effect of search difficulty was significant, with no main effect or interactions involving speaker group. ${ }^{3}$

\footnotetext{
${ }^{2}$ Set size was sampled in equal proportions across all conditions. There were no reliable Set Size $\times$ Speaker Group interactions for overall search RTs, first fixation times, or decision times. In post-hoc comparisons, there were no reliable differences between bilinguals and monolinguals for any set size. In the interest of brevity, we avoid reporting set size effects or interactions. Due to the high number of conditions in Experiment 1 (trial type, search difficulty, speaker group, and set size), there were too few trials per condition for a 2.5$S D$ cutoff criterion to be applied.

Due to occasional missing eye movement data, the overall search RTs do not equal the sum of the first fixations times and decision times.

${ }^{3}$ In addition to first fixation times, oculomotor control was also measured using scan path ratios, which are the summed amplitudes of all saccades (in degrees of visual angle) prior to target fixation, divided by the shortest possible distance between the central fixation and the target (e.g., Hout \& Goldinger, 2015). The analyses revealed no reliable scan path ratio differences between speaker groups in any experiment.
} 


\section{Decision times}

As with first fixation times, the main effect of speaker group was not significant. The Search Difficulty $\times$ Speaker Group interaction was marginal $(p=.055)$ in the black condition (slightly favoring the bilingual group), and was null in the color condition.

\section{Bayes analysis}

We computed scaled Jeffrey-Zellner-Siow (JZS) Bayes factor values for speaker group across the three experiments (see Appendix Table 9). This analysis shows moderately strong evidence in favor of the null hypothesis (about 3:1) in nearly all comparisons, and a monolingual advantage in one comparison.

\section{Language balance}

To assess whether bilingual language experience predicted visual search performance, language balance (calculated as ratio of average proficiency in the second and first language) was used as a predictor in simple linear regression analyses. Language balance did not predict overall search RTs, first fixation times, or decision times in either color condition at any level of search difficulty (all $p \mathrm{~s}>.05$ ).

\section{Discussion}

In Experiment 1, bilinguals and monolinguals performed visual search under varying degrees of difficulty. The findings were consistent with previous studies with regard to search difficulty (e.g., Duncan \& Humphreys, 1989), but there were no RT differences between bilinguals and monolinguals in any condition. Additionally, search RTs did not vary as a function of language balance in bilinguals. These results contrast with previous findings of an overall bilingual advantage in visual search (e.g., Friesen et al., 2015; Hernández et al., 2012). There was also no consistent evidence of a bilingual advantage in the eye movement data: Search guidance, as measured by first fixation times, was equivalent for both groups.

Interestingly, bilinguals had marginally faster decision times than monolinguals in the most difficult black condition, but no group differences were observed in the color condition. This may indicate that a bilingual advantage in visual search occurs at the decision level, when the target template held in memory is matched to the visual stimulus. Bialystok, Craik, and Ruocco (2006) found that bilinguals were faster than monolinguals on an antisaccade task when the participants were required to respond using a keypress. The authors argued that bilingualism may affect the symbolic mapping between a visual stimulus and the appropriate response. This marginal difference may be more robust when decisions are made more difficult.

\section{Experiment 2}

The marginal group difference in decision times observed in Experiment 1 suggests that, if there is a bilingual advantage in visual search, it may occur at the decision-making stage, rather than during attentional guidance. To examine this possible advantage more closely, we modified the task to increase the difficulty of the decision. Participants performed visual search, during which they reported the color or shape of geometric figures embedded within target Landolt Cs. In some blocks, participants only reported the color or shape of the embedded geometric figure. In other blocks, they alternated between color and shape decisions.

Previous researchers have reported bilingual advantages in similar switching tasks. Prior and MacWhinney (2010) compared bilinguals and monolinguals in a switching task wherein participants were shown red or green circles and triangles, and were asked to report either the color or shape. Performance was measured by examining switching and mixing costs in RTs. Switching costs were calculated by comparing switch and repeat trials in a switching-only condition. "Switch" trials were defined as a "shape" decision following a "color" decision, or vice versa. Mixing costs were calculated by comparing repeat trials in the switching condition to repeat trials in color-only or shape-only conditions. The authors found that bilinguals showed reduced switching costs, relative to monolinguals, but equivalent mixing costs. They suggested that lifelong bilingual experience contributed to task switching advantages. Additionally, Prior and Gollan (2011) observed that bilinguals who reported more frequent language switching showed reduced switching costs, as compared both to monolinguals and to bilinguals who reported less frequent language switching. The authors proposed that daily language habits may shape general cognitive mechanisms that play a role in nonverbal tasks (see also Soveri, Rodriguez-Fornells, \& Laine, 2011). If bilinguals have an advantage over monolinguals in both task switching and decision efficiency under difficult visual search conditions, they should show faster decision times, particularly in the switching condition.

\section{Method}

\section{Participants}

Nineteen English monolingual speakers and 18 bilingual speakers participated in Experiment 2. No volunteers had participated in Experiment 1. Recruitment procedures were identical to Experiment 1. Bilingual speakers reported speaking English and one the following languages: Spanish (9), French (2), Korean (2), Mandarin (1), Arabic (1), Navajo, (1), Serbian (1), and Vietnamese (1). The monolingual group had ten females, a mean age of 18.6 years $(S D=0.90)$ and mean education of 13.3 years $(S D=0.56)$. The bilingual group had 11 females, a mean age of 19.1 years $(S D=1.74)$ and mean education of 13.2 years 
( $S D=0.57)$. The groups did not differ in age, education, or maternal education (all $t \mathrm{~s}<1.18$ ).

\section{Stimuli}

The targets were Landolt Cs with gaps on the top, bottom, left, or right. All targets and distractors were black. Embedded within each Landolt $\mathrm{C}$ was a triangle or square shape, which was either solid black (filled) or an outline of the shape (unfilled). Stimuli were presented in the easy (100\% circle distractors), medium (50\% circle distractors, $50 \%$ Landolt $\mathrm{C}$ distractors), and difficult (100\% Landolt C distractors) conditions.

\section{Procedure}

Each participant was familiarized with the shape and color judgments prior to the visual search trials. In each practice trial, either the word "shape" or "color" was shown, along with a reminder of the response keys, followed by a 1,000$\mathrm{ms}$ fixation cross and a single Landolt $\mathrm{C}$ in the center of the screen, containing an embedded shape. In shape trials, the participant quickly judged whether the shape was a triangle or a square by pressing the "a" or "d" keys, respectively, while ignoring the color of the shape. Responses had to be made within $5 \mathrm{~s}$ or the trial was terminated. In color trials, the participant judged whether the embedded shape was unfilled or filled by pressing the " $\mathrm{j}$ " or "l" keys, respectively, while ignoring the particular shape. Participants rested their index and middle fingers of each hand on these keys throughout the experiment. The practice session consisted of 20 color judgment trials, 20 shape judgment trials, and a block of 40 switch trials, in which color and shape judgments were randomly intermixed. The practice switching block was repeated if the participant did not achieve $80 \%$ accuracy.

The experimental trials were similar to the practice trials, with the addition of a visual search component (see Fig. 2 for a schematic trial). At the onset of each trial, the participant was shown the target Landolt $\mathrm{C}$ without an embedded shape, along with the word "shape" or "color," indicating the judgment required for that trial. The subsequent visual search display (preceded by a 500-ms fixation cross) contained 24 stimuli, each with an embedded shape. One of the shapes was the target. Participants were instructed to quickly locate the target and make the appropriate "shape" or "color" judgment. The trial terminated when any response key was pressed (or when $15 \mathrm{~s}$ had elapsed). After completing 24 practice trials, participants completed three blocks each of color-only, shape-only, and switch trials, and each block contained either easy, medium, or difficult trials. Each single-task block (color or shape) consisted of 20 trials, whereas the switch blocks consisted of 40 trials. The eyetracking apparatus was the same as in Experiment 1, and the same measurements were collected. After completing the search portion of the experiment, participants completed symmetry span and RAPM in counterbalanced order across participants.

\section{Results}

We again focus on the main effects and interactions involving speaker group, since these were the analyses of interest.

\section{Normative measures}

There were no reliable group differences between bilinguals and monolinguals on either the symmetry span task or RAPM (both $t \mathrm{~s}<1.3$ ).

\section{Visual search}

RTs slower than 2.5 SDs from individual participant means were removed from analyses $(2.7 \%$ of the data). Another 2.5-SD cutoff was used to remove outliers from the first fixation times and decision times $(2.4 \%$ of the data). A 3 (Decision Block: color, shape, or switch) $\times 3$ (Search Difficulty: easy, medium, or difficult) $\times 2$ (Speaker Group: monolingual or bilingual) mixed ANOVA was conducted on the mean correct RTs (see Appendix Table 4 for all ANOVA results, including effect sizes). All post-hoc pairwise comparisons were evaluated using a Bonferroni-corrected alpha of .017 for simultaneous comparisons. Figure 3 shows all the visual search results, with overall RTs in panel A, first fixation times in panel $\mathrm{B}$, and decision times in panel $\mathrm{C}$ (see also Appendix Table 7).

Mixing costs and switching costs were also analyzed. Switching costs reflect the difference between switch trials (from shape to color, or vice versa) and repeat trials in the switch condition. Mixing costs reflect the difference between repeat trials in the switch condition and responses in the single-decision conditions (see Prior \& Gollan, 2011; Prior $\&$ MacWhinney, 2010). Mixing costs were calculated by subtracting the average RT for repeat trials in the singledecision conditions (color and shape) from the average RT for repeat trials in the switch condition. Costs were analyzed using a 2 (Cost Type: switching or mixing) $\times 3$ (Search Difficulty: easy, medium, or difficult) $\times 2$ (Speaker Group: monolingual or bilingual) mixed ANOVA.

\section{Overall search $R T$}

The main effect of decision block was reliable: RTs in the switch condition were slower than RTs in the color $[t(36)=$ $3.25, p=.003]$ and shape $[t(36)=2.72, p=.010]$ conditions. The main effect of speaker group was null. The three-way interaction was significant $(p=.038)$; however, none of the 


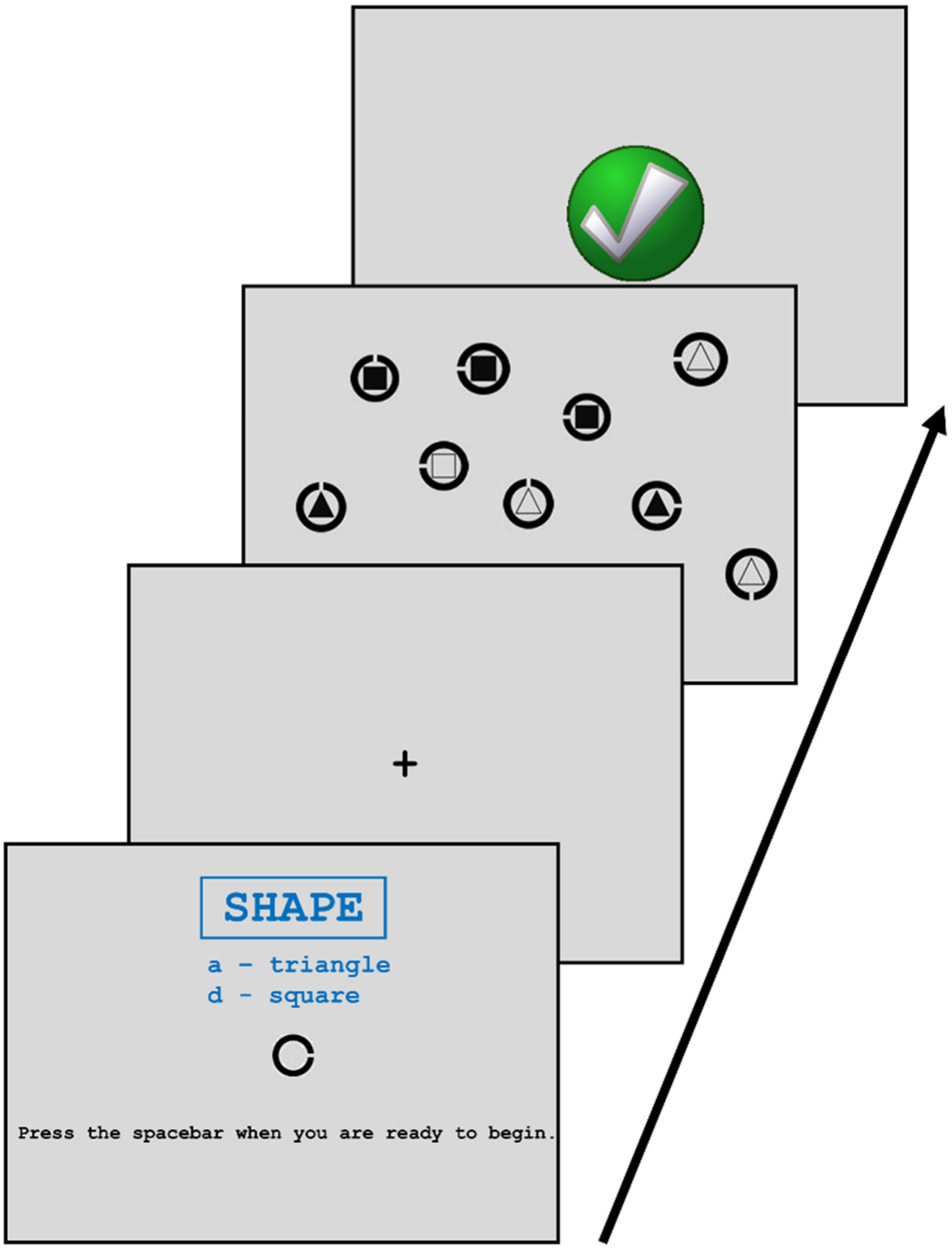

Fig. 2 Progression of trial events in Experiment 2 


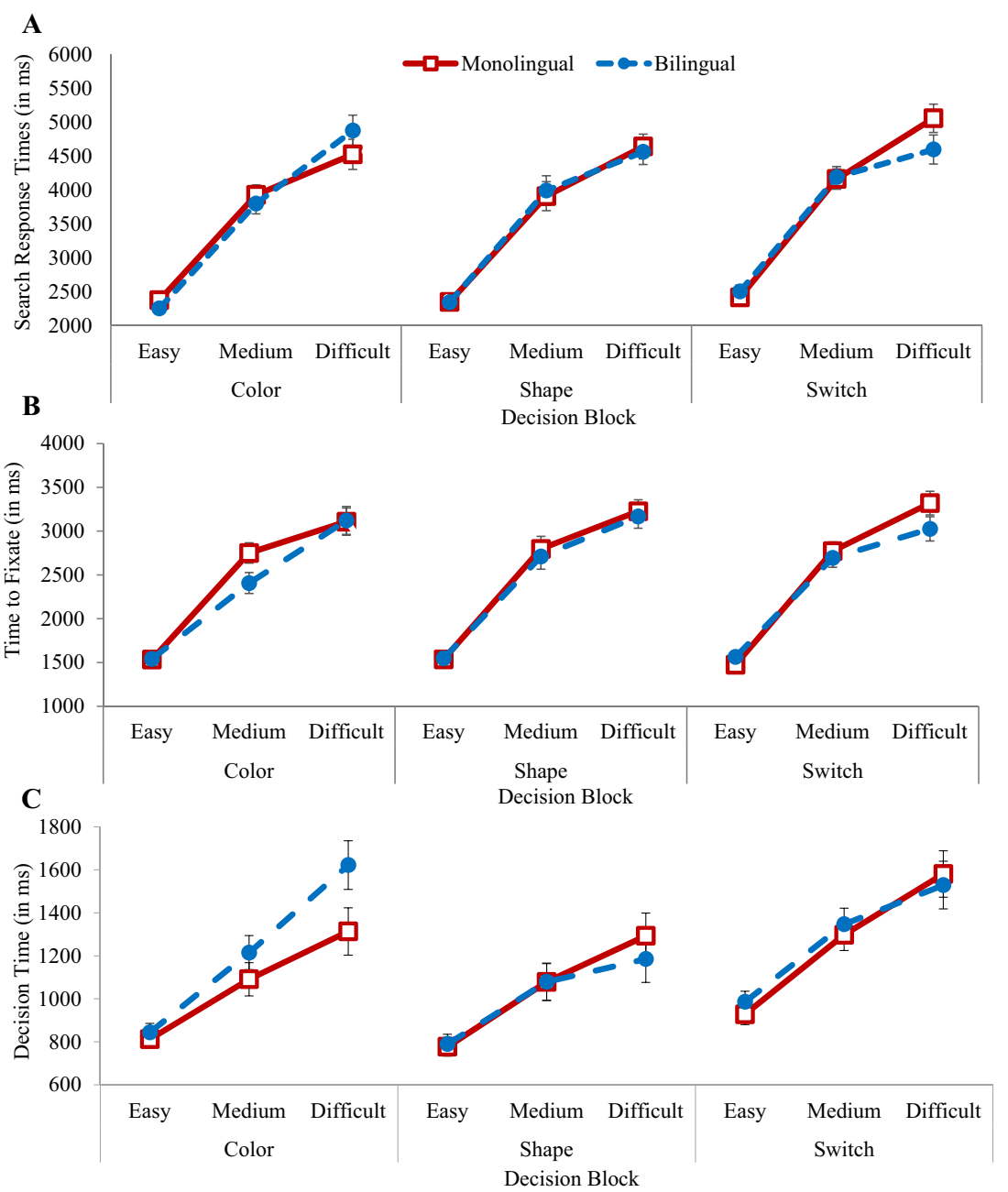

Fig. 3 Results from Experiment 2. (A) Mean overall search RTs. (B) Mean first fixation times. (C) Mean decision times. Error bars show \pm 1 SEM

post-hoc comparisons were reliable. No other interactions were reliable. $^{4}$

Figure 4 shows switching costs (panel A1) and mixing costs (panel A2) in overall search RTs. The main effect of cost type was reliable $\left[F(1,35)=4.56, p=.040, \eta_{\mathrm{p}}{ }^{2}=.044\right]$ : Mixing costs $(M=207 \mathrm{~ms})$ were greater than switching costs $(M=-34 \mathrm{~ms})$. The main effect of speaker group was not significant $(F<1)$, and the three-way interaction was marginal $\left[F(2,70)=3.12, p=.051, \eta_{\mathrm{p}}{ }^{2}=.082\right]$. Monolinguals had smaller switching costs than bilinguals in the medium and difficult search conditions, but these differences were not reliable. Bilinguals had marginally smaller mixing costs in the

\footnotetext{
$\overline{{ }^{4} \text { An additional }} 40$ bilinguals and 41 monolinguals completed the behavioral portion of the experiment. For these participants, we found a significant main effect of decision block $\left[F(2,158)=45.89, p<.001, \eta_{p}{ }^{2}=.367\right]$ on overall search RTs. There was also a significant three-way Condition $\times$ Search Difficulty $\times$ Speaker Group interaction $\left[F(4,316)=2.53, p=.041, \eta_{\mathrm{p}}{ }^{2}=\right.$ $.031]$, but no post-hoc comparisons were reliable. In the cost analysis, the Search Difficulty $\times$ Speaker Group interaction was significant $[F(2,158)=$ $\left.3.27, p=.041, \eta_{\mathrm{p}}{ }^{2}=.040\right]$; however, no post-hoc comparisons were reliable. No other main effects or interactions were reliable.
}

difficult search condition $[t(35)=2.44, p=.020]$. No other interactions were significant.

\section{First fixation times}

The main effect of speaker group was not reliable (see Fig. 3B), nor were any interactions. For the cost analysis, the main effect of cost type was marginal $[F(1,35)=3.92, p=$ $\left..056, \eta_{\mathrm{p}}{ }^{2}=.101\right]$, with mixing costs $(M=69 \mathrm{~ms})$ being slightly greater than switching costs $(M=-114 \mathrm{~ms}$; see Fig. 4, panels $\mathrm{B} 2$ and $\mathrm{B} 1$, respectively). No other effects or interactions were reliable (all $\left.F_{\mathrm{S}}<1.79\right)^{5}$

\footnotetext{
${ }^{5}$ During the initial portion of the visual search task, reflected by the first fixation times, participants are scanning the screen for a target item. It is unlikely that the switching or mixing costs affected this part of the task. Thus, these graphs should be interpreted with caution. The switching and mixing costs are most accurately reflected in decision times.
} 
$\rightarrow$ Monolingual $\longrightarrow-$ Bilingual
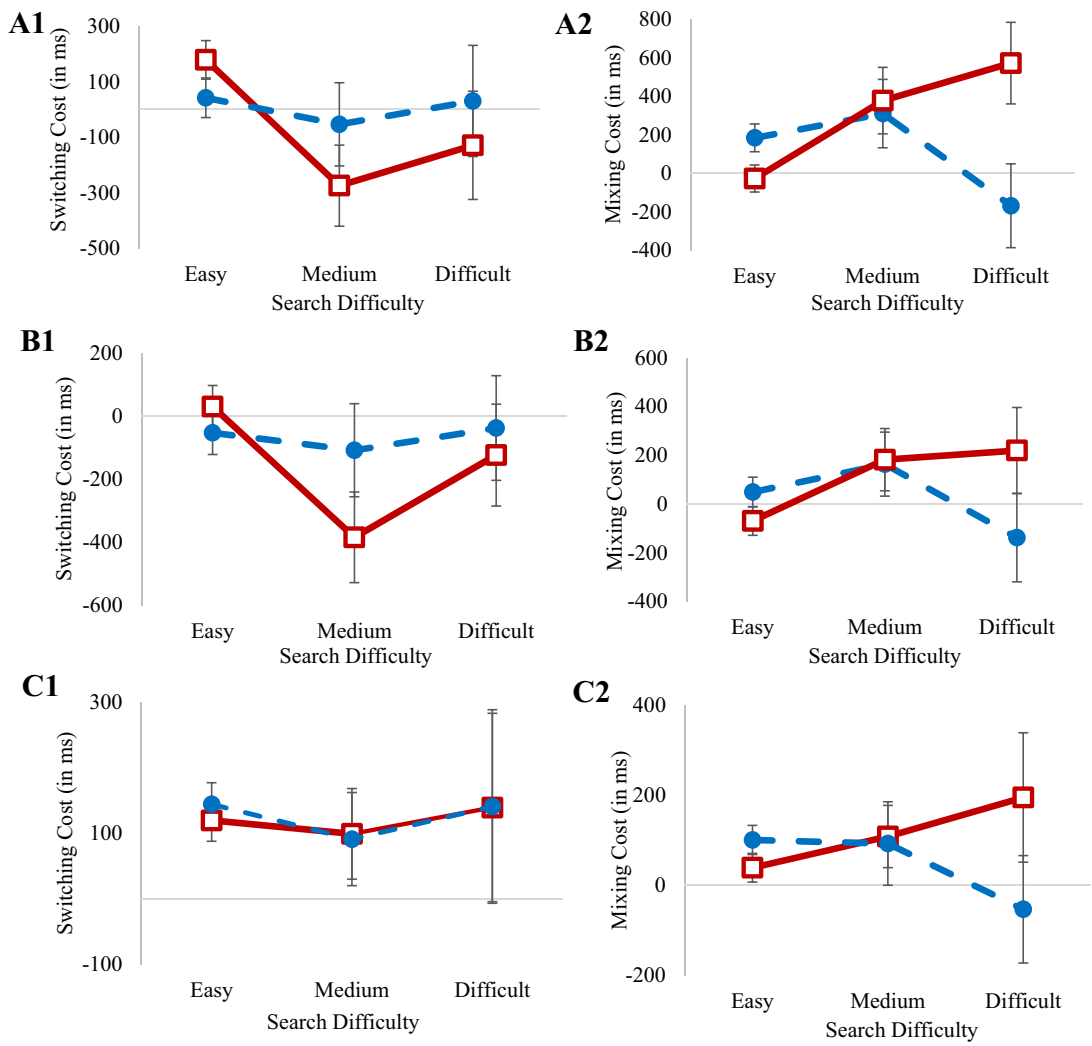

Fig. 4 Results from Experiment 2. (A) Overall search RTs: Switching costs (A1) and mixing costs (A2). (B) First fixation times: Switching costs (B1) and mixing costs (B2). (C) Decision times: Switching costs (C1) and mixing costs (C2). Error bars show \pm 1 SEM

\section{Decision times}

The results from the decision time analyses are depicted in Fig. 3C. The main effect of decision block was reliable, with slower decision times in the switch than in the shape condition $[t(36)=5.27, p<.001]$, as well as slower decision times in the color than in the shape condition $[t(36)=4.56, p<.001]$. Decision times were not significantly different in the color and switch conditions $(t<1.55)$. The main effect of speaker group was null, and no interactions were reliable. In the cost analysis (shown in Fig. 4C), no main effects or interactions were significant.

\section{Language balance}

As in Experiment 1, linear regression analyses were conducted for the bilingual group using language balance as a predictor variable. Language balance was not a reliable predictor of overall search RTs in any condition (all $p s>.05$ ), although it was a marginally significant predictor of first fixation times in the switching condition under difficult visual search $(\beta=-.468)$ $\left[t(17)=-2.12, p=.050, R_{\text {Adj }}^{2}=.170\right]$. Language balance was a reliable predictor of decision times in the color condition under medium search difficulty $(\beta=-.505)[t(17)=-2.34, p=.032$, $\left.R_{\text {Adj }}^{2}=.209\right]$, and in the shape condition under difficult search $(\beta$ $=-.494)\left[t(17)=-2.27, p=.037, R_{\text {Adj }}^{2}=.197\right]$, but it did not predict switching or mixing costs for any measure (all $p \mathrm{~s}>.10$ ).

\section{Discussion}

As expected, search performance decreased as difficulty increased, particularly under task switching conditions. However, no reliable visual search differences emerged between bilinguals and monolinguals in the behavioral or eyetracking measures. The marginal group difference in decision times observed in Experiment 1 was not replicated in Experiment 2. Indeed, bilinguals showed a slight disadvantage in decision times in the difficult color task condition. Overall, the RT and eye movement results argue against a bilingual advantage in task switching during visual search (in particular decision times). Only one important trend was observed in the proficiency analyses: Balanced bilinguals, as compared to bilinguals who use one language predominantly, had faster decision times in two measures (in the medium difficulty color condition and the difficult shape condition). However, this trend was not consistent across conditions.

Previous studies have reported bilingual advantages in switching costs (e.g., Prior \& Gollan, 2011; Prior \& MacWhinney, 2010). In Experiment 2 we observed a marginal bilingual advantage, but only in mixing costs and overall search RTs, not in decision times or first fixation times. Additionally, 
there was a bilingual disadvantage in switching costs. Given the inconsistent effects across measures, these results do not support a bilingual advantage in task switching.

\section{Experiment 3}

Experiments 1 and 2 provided no clear evidence of a bilingual advantage in attentional guidance or decision-making processes. Bilinguals were largely indistinguishable from monolinguals in their visual search performance, whether they were required to inhibit irrelevant information (Exp. 1) or to switch attentional sets (Exp. 2). However, bilinguals might still demonstrate a cognitive advantage over monolinguals in their ability to update and/or continuously monitor information held in working memory. Experiment 3 was conducted to investigate this possibility.

Previous studies have suggested that bilinguals show dualtask advantages (e.g., Bialystok, Craik, \& Ruocco, 2006). In Experiment 3, we investigated whether bilinguals and monolinguals differed in the monitoring of information and the updating of items held in working memory within the context of a visual search task. Bialystok, Craik, and Ruocco investigated updating and monitoring using a dual-modality task with both auditory and visual stimuli. Their participants simultaneously sorted auditory and visual items into categories (letters/numbers and animals/musical instruments), using a keypress for visual items and verbal responding for auditory items. Bilinguals correctly sorted more items than monolinguals, suggesting superior updating and monitoring ability. However, other researchers have failed to observe a bilingual advantage in dual-task performance. Moradzadeh, Blumenthal, and Wiseheart (2015) investigated bilinguals' dual-task performance on a Krantz (2007) task and a dual nback task. In the Krantz task, participants tracked a moving dot while simultaneously attending to a series of flashing letters. In the dual $n$-back task, participants simultaneously reported whether a visual stimulus (e.g., colored square) and an auditory stimulus (e.g., digit) matched a previously presented visual or auditory stimulus. Moradzadeh et al. failed to observe a bilingual advantage in either task.

Following these studies, the participants in Experiment 3 performed a dual task within the visual search paradigm, which required the target stimuli to be continuously updated. Participants mentally rotated a target Landolt $\mathrm{C}$ for the search task, remembered an abstract shape for a memory task, or simultaneously performed both tasks. This design enabled us to investigate whether a bilingual advantage might take the form of better updating of memorial representations, better ability to hold an image in memory, or a combination of both. Previous results (e.g., Bialystok, Craik, \& Ruocco, 2006) suggested that a bilingual advantage in updating should be observed in the most demanding condition (i.e., dual monitoring).

\section{Method}

\section{Participants}

Twenty-four new English monolingual speakers and twentynine new bilinguals participated in the experiment. The recruitment procedures were identical to those of Experiment 1. Bilingual speakers reported speaking English and one of the following languages: Spanish (11), Mandarin (7), Hindi (2), or other (9). The monolingual group featured 16 females, a mean age of 18.75 years $(S D=0.79)$, and mean education of 13.7 years $(S D=0.82)$. The bilingual group featured $18 \mathrm{fe}$ males, a mean age of 20.76 years $(S D=3.03)$, and mean education of 14.0 years $(S D=2.76)$. The groups did not differ in either education or maternal education (both $t \mathrm{~s}<1.65$ ), but the bilinguals were significantly older than the monolinguals $[t(51)=3.15, p=.003]$.

\section{Stimuli}

Targets consisted of Landolt Cs with gaps on the top, bottom, left, or right. All targets and distractors were black. Novel shape (polygon) stimuli were taken from Tat and Azuma (2016), with each subtending approximately $2^{\circ} \times 2^{\circ}$ (centered). We created three stimulus conditions: easy (50\% of the distractors were circles and 50\% were Landolt Cs), medium $(25 \%$ circles and $75 \%$ Landolt Cs), and difficult (100\% Landolt Cs).

\section{Procedure}

The visual search task was administered across four conditions: (1) only visual search, (2) visual search with updating, (3) visual search with secondary memory task, and (4) visual search with updating and secondary memory task.

Updating task The updating component required participants to mentally update a target Landolt $\mathrm{C}$ over successive trials. In trial $n$, the target $\mathrm{C}$ was shown. In trial $n+1$, the target was removed, and participants had to mentally rotate the target counterclockwise 90 degrees from the previous trial. After incorrect search trials, participants were reminded of the current target (these trials were discarded from the analysis).

Secondary memory load In the secondary-load component, participants remembered a polygon shown prior to each visual search trial. After completing the search task, a final screen with four polygon alternatives was displayed, and participants selected the correct polygon by pressing the "d," "f," " $\mathrm{j}$," or 
"k" key. The final screen was shown until the participant responded (or until $10 \mathrm{~s}$ had elapsed).

In all conditions, an initial screen depicted a polygon prior to search, and a final screen depicted four polygons after the search. In conditions without the secondary task, participants were told simply to ignore the polygons. The initial screen was shown for a maximum of $10 \mathrm{~s}$ or until the space bar was pressed. Following a gaze-contingent 500 -ms fixation cross, the participants were shown the Landolt $C$ target cue until they pressed the space bar to begin the search. Note that in conditions that included the updating task, the target cue was not displayed after correct updating trials. In all search trials there were 24 items, including the target, which was presented in a random location. The search display was terminated when the participants pressed the space bar (or after $15 \mathrm{~s}$ had elapsed). After the space bar was pressed, the stimuli were replaced by numbers for $1,000 \mathrm{~ms}$. The participants indicated the target location by selecting its number from two alternatives shown in a subsequent probe screen. After search feedback was presented, the final screen of polygons was displayed (for conditions without the secondary task, this screen was displayed for 2,000 ms). See Fig. 5 for a sample trial progression.

Participants completed one block apiece of easy, medium, and difficult trials across each of the four task conditions, for a total of 12 randomly ordered blocks with 16 trials each. Participants also completed six practice trials for each condition, for a total of 24 practice trials. Each participant was required to attain $80 \%$ accuracy in each practice condition, or the condition would restart until this criterion was satisfied. After completing the search tasks, participants completed the symmetry span and RAPM tasks, with the order counterbalanced across participants.

\section{Results}

We again focus on the main effects and interactions involving speaker group, since these were the analyses of interest.

\section{Normative measures}

We found no reliable group differences between bilinguals and monolinguals on either the symmetry span task or RAPM (both $t \mathrm{~s}<1$ ).

\section{Visual search}

Search RTs slower than 2.5 SDs from the individual participant means were removed from the analyses (2.9\% of the data). Another 2.5-SD cutoff was used to remove outliers from the first fixation times and decision times (2.8\% of the data). A 4 (Updating Condition: control, updated search, secondary load, or dual task) $\times 3$ (Search Difficulty: easy, medium, or difficult) $\times 2$ (Speaker Group: monolingual or bilingual) mixed ANOVA was conducted on the mean correct RTs (see Appendix Table 5 for all ANOVA results, including effect sizes). Post-hoc pairwise comparisons were evaluated using Bonferroni-corrected alphas of .017 or .01, for three or six simultaneous comparisons, respectively. Figure 6 shows all visual search results, with overall RTs in panel A, first fixation times in panel B, and decision times in panel C (see also Appendix Table 8).

\section{Overall search $R T$}

The main effect of updating condition was significant: RTs in the dual-task condition were slower than those in the control $[t(50)=7.05]$, updated search $[t(52)=4.50]$, and secondaryload $[t(49)=5.88]$ conditions (all $p \mathrm{~s}<.001$ ). Additionally, the updated-search condition RTs were slower than both the control condition RTs $[t(51)=3.77, p<.001]$ and the secondaryload condition RTs $[t(59)=2.90, p=.006]$. Although the main effect of speaker group was not significant, there was a significant Updating Condition $\times$ Speaker Group interaction (see Fig. 6A). Bilinguals were slower than monolinguals in the dual-task condition ( 4,802 vs. $4,338 \mathrm{~ms})$ and in the updatedsearch condition ( 4,411 vs. $3,720 \mathrm{~ms}$ ), but were slightly faster in the control condition ( 3,275 vs. $3,482 \mathrm{~ms})$. The groups were statistically equivalent in the secondary-load condition $(t<1)$. The Search Difficulty $\times$ Speaker Group interaction was significant, but post-hoc comparisons were not statistically reliable. No other interactions were reliable.

\section{First fixation times}

There was a significant main effect of updating condition: First fixations to targets were slowest in the dual-task condition, relative to the other three conditions [all ts $(51)>3.90, p<$ $.001]$. We observed no reliable effect of speaker group, nor any reliable interactions (see Fig. 6B).

\section{Decision times}

The main effect of updating condition was significant, with slower decision times in the dual-task condition, relative to all others (all $t \mathrm{~s}>3.2, p<.01$ ). Additionally, updated-search condition decision times were slower than control and secondary-load condition decision times $[t(53)=5.04$ and 5.44, respectively, $p$ s $<$ $.001]$. The main effect of speaker group was robust: Bilinguals had significantly slower decision times than monolinguals. The three-way interaction was also reliable, with differences between the groups varying across conditions and difficulty levels (see Fig. 6C).

\section{Secondary task performance}

In the secondary-load and dual-task conditions, bilinguals and monolinguals did not differ in secondary-task accuracy (both $t \mathrm{~s}$ 


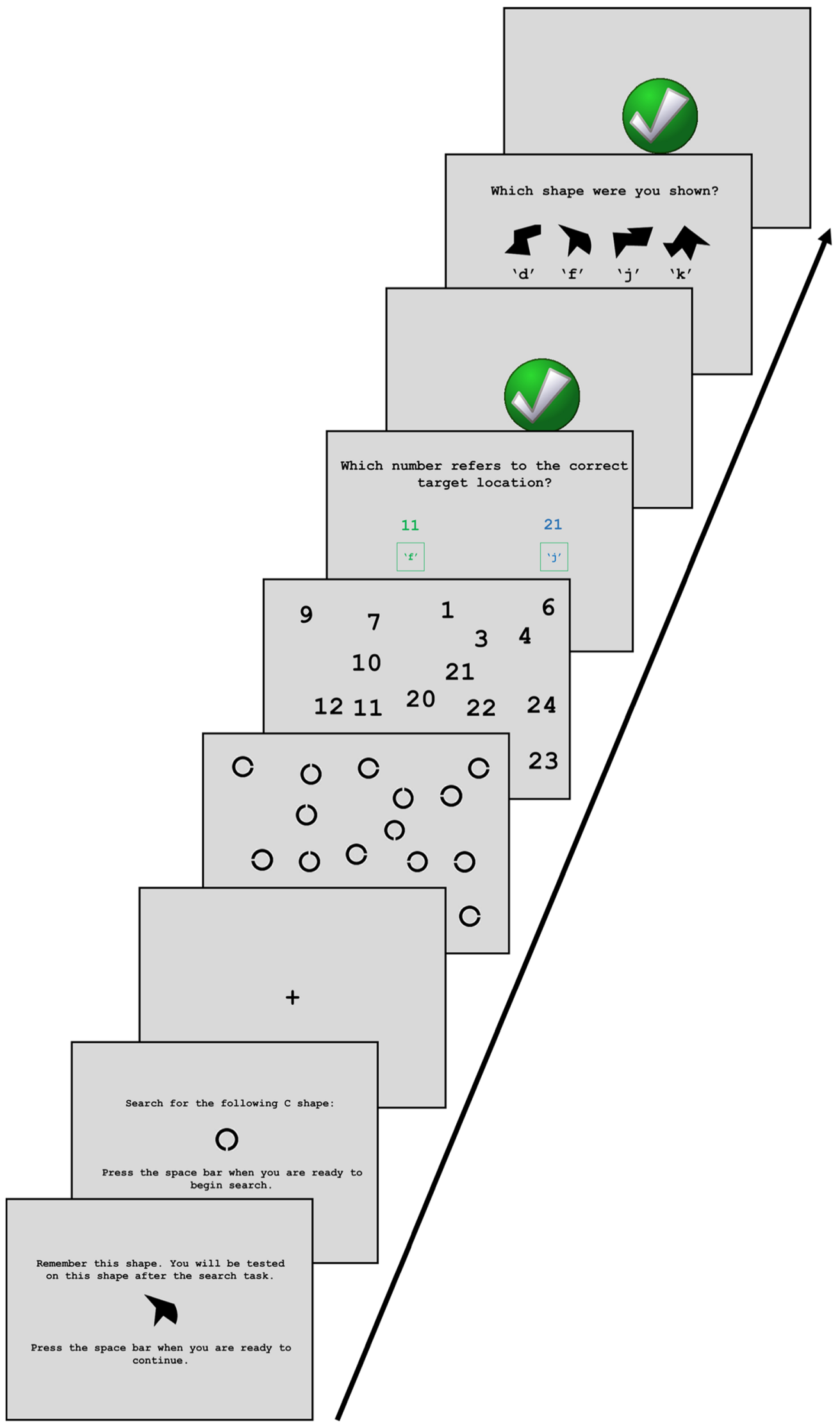

Fig. 5 Progression of trial events in Experiment 3, depicting the dual-task condition 


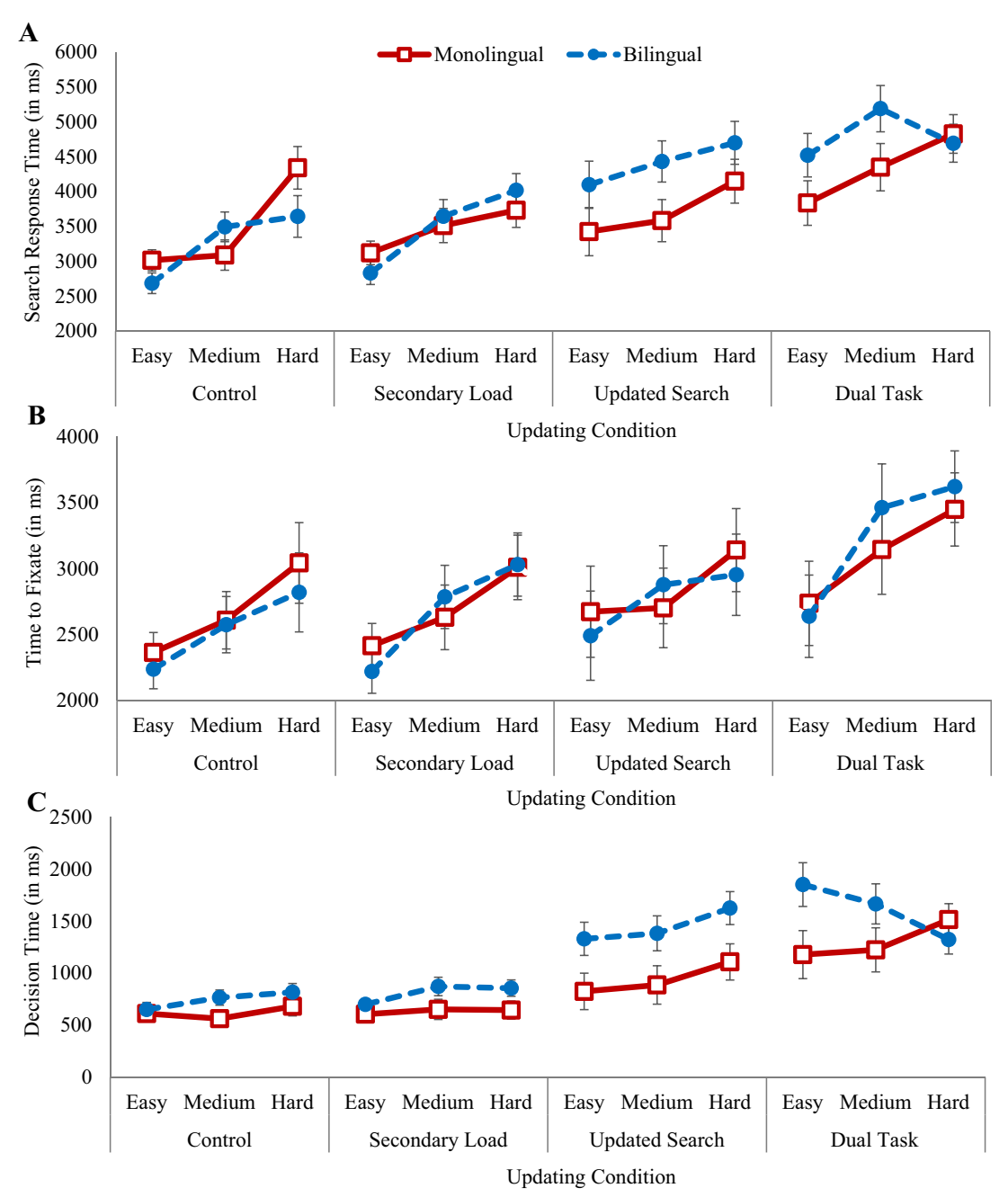

Fig. 6 Results from Experiment 3. (A) Mean overall search RTs. (B) Mean first fixation times. (C) Mean decision times. Error bars represent \pm 1 SEM

$<1)$. Accuracy for the polygon memory task also did not differ between the secondary-load and dual-task conditions $(t<1)$.

\section{Language balance}

As in Experiments 1 and 2, linear regression analyses were conducted for the bilingual group using language balance as a predictor variable. Language balance significantly predicted overall search RTs in the control condition under easy search $(\beta=.459)$ $\left[t(28)=2.68, p=.012, R_{\text {Adj }}^{2}=.181\right]$, as well as first fixation times in the medium difficulty condition $(\beta=.393)[t(27)=2.18$, $\left.p=.038, R_{\text {Adj }}^{2}=.122\right]$. Additionally, language balance was a reliable predictor of decision times in the control condition under easy search $(\beta=-.387)\left[t(28)=-2.18, p=.038, R_{\text {Adj }}^{2}=.118\right]$. No other regression analyses were reliable.

\section{Discussion}

As expected, visual search was slower with increased task difficulty. Search RTs confirmed that the dual-task condition was most difficult, followed by updated search, secondary load, and control. As in Experiments 1 and 2, no bilingual advantage in search RTs was observed for any condition. It was hypothesized that any bilingual dual-task advantage should be most evident in the conditions with the greatest cognitive demands (e.g., the dual-task/difficult condition). However, the bilingual and monolingual groups performed equivalently in the easy and difficult conditions, and bilinguals were marginally slower than monolinguals in the medium condition. Additionally, when participants had to mentally update targets, bilinguals were generally slower than monolinguals, a difference reflected in increased decision times. Eye movement analyses revealed no evidence of a bilingual advantage in search guidance (first fixation time) or response efficiency (decision time). Indeed, the most reliable group difference was a bilingual disadvantage in decision times. These findings argue against a bilingual advantage in visual search and dual-task performance, and are inconsistent with previous reports of a bilingual advantage, particularly under conditions of high monitoring (e.g., Costa et al., 2009), but are 
consistent with the findings of Moradzadeh et al. (2015), who also failed to observe a bilingual dual-task advantage.

\section{General discussion}

The present experiments used nonverbal visual search paradigms to explore possible bilingual advantages in visual search, across different levels of search difficulty and under various cognitive demands. Previous investigations of the purported bilingual advantage in visual search have been inconsistent; however, these studies mainly employed relatively coarse-grained dependent measures such as RT and accuracy. Such measures may not be sufficiently sensitive to reveal subtle group differences. We examined fine-grained eyetracking measures, which allowed us to assess whether bilinguals and monolinguals differed in attentional guidance or in perceptual decision-making. Each experiment recruited a distinct set of cognitive demands in order to broadly investigate the conditions under which a bilingual advantage may exist: Experiment 1 was conducted to investigate whether bilinguals exhibit an enhanced ability to inhibit irrelevant visual information, by presenting feature and conjunction search tasks across different difficulty levels. Experiment 2 examined whether bilingualism confers a benefit in the ability to switch tasks when making decisions, by requiring participants to alternate between different attentional sets throughout the visual search session. Experiment 3 was conducted to test for a potential bilingual advantage in the ability to continuously monitor and update information, by requiring participants to mentally rotate their internal visual target templates while simultaneously maintaining an unrelated abstract image in memory.

Across all three experiments, there was no clear evidence of a bilingual advantage in visual search. Our eye movement analyses revealed no bilingual advantage in measures of search guidance (i.e., first fixation times). Although bilinguals showed a slight benefit over monolinguals in task switching ability in one condition of Experiment 2 (i.e., reduced costs in "repeat" trials that were intermixed with "switch" trials), this finding is inconsistent with previous studies: Previous research has shown a bilingual advantage in task switching costs (i.e., the cost of switching types of decisions in consecutive trials), but not in mixing costs (Prior \& MacWhinney, 2010). Prior and MacWhinney suggested that mixing costs are associated with sustained activation of goal maintenance, which is required when potential responses compete with each other and is associated with updating of mental representations (e.g., Miyake et al., 2000). If the slight bilingual advantage in mixing costs observed in the present study reflected better goal maintenance, this same advantage should have arisen in Experiment 3, which tested the ability to continuously maintain and update relevant visual information. Given that no bilingual advantage was obtained in Experiment 3, the finding of reduced mixing costs for bilinguals was likely more reflective of task-specific differences between groups, rather than differences in goal maintenance and updating ability.

Notably, although our bilingual sample was sufficiently representative, we observed no clear bilingual advantages, despite using tasks designed to place varying demands on executive function and working memory. Our sample was comparable to bilingual samples from similar studies (e.g., Chabal et al., 2015; Friesen et al., 2015; Hernández et al., 2012). Participants also reported acquiring both languages in childhood (with the vast majority acquiring both languages by the teenage years), with high levels of proficiency in both languages across multiple modalities, and daily use of each language, consistent with the criteria cited by Bialystok (2009). Despite using these wellestablished criteria to recruit bilingual participants, we acknowledge that our sample was somewhat heterogeneous. Although heterogeneity may increase the generalizability of the results (Friesen et al., 2015), it may have contributed to the null results in our study and to other conflicting findings regarding the bilingual cognitive advantage in young adults (e.g., Bialystok, 2006; Kousaie \& Phillips, 2012; Paap \& Greenberg, 2013; Ratiu \& Azuma, 2015). However, several visual search studies have reported such an advantage with college-aged participants like those in our present samples (e.g., Friesen et al., 2015; Hernández et al., 2012).

Another possible explanation for our null results could be our relatively small sample sizes. Perhaps our data contained trends toward a bilingual advantage, but we lacked the statistical power to classify such trends as reliable. The small sample sizes may have contributed to larger standard deviations in some conditions making small or subtle group differences harder to detect. To address these concerns, we include Appendix Figs. 8, 9, 10, 11, 12, 13, 14, 15, 16, 17, 18 and 19 , which shows scatterplots of individuals' performance across all conditions. These plots show that the bilingual and monolingual groups consistently overlap, and therefore that increased sample sizes likely would not change these trends. Indeed, when we recruited larger samples sizes (e.g., Exp. 3), we found no reliable differences between groups. To further examine these null data, we calculated scaled JZS Bayes factor values (see Appendix Table 9). These analyses provide an odds ratio in favor of the null or alternative hypothesis. The results show moderately strong evidence in favor of the null hypothesis (about 3:1) in nearly all comparisons (Rouder, Speckman, Sun, Morey, \& Iverson, 2009). Post-hoc power analyses were conducted to determine the appropriate sample sizes needed to detect differences between bilinguals and monolinguals (see Appendixes Tables 3, 4 and 5). Over 100 participants would be needed to detect an effect of speaker group in any condition, which reflects the absence of any robust underlying group differences, and greatly exceeds standard sample sizes in the literature.

One finding that may have implications for future studies of bilingual cognition is that individual differences in 
language experience (language balance) predicted performance across several conditions in Experiments 2 and 3. This result is consistent with previous research suggesting that bilinguals' language-switching experience may influence executive function (Prior \& Gollan, 2011). Bilinguals who use both languages daily must switch between languages on a more regular basis than less-balanced bilinguals, and this experience may lead to differences in how they perform nonlinguistic tasks. However, this effect should be interpreted with caution, given that it was not observed across all experiments.

Overall, the results of the present study did not reveal a robust bilingual advantage in visual search. If being bilingual or multilingual confers attentional, executive function, or working memory capacity advantages, a consistent difference should have been observed across the present experiments. The only reliable group difference was observed in Experiment 3, in which bilinguals exhibited a disadvantage in search decision times relative to monolinguals. We observed no apparent differences between monolinguals and bilinguals in attentional guidance, response initiation, or overall search performance. Although bilinguals did not show a consistent visual search advantage relative to monolinguals, the possibility still exists that specific aspects of the bilingual experience may be related to search performance (e.g., Friesen et al., 2015; Hernández et al., 2012). Examining the contribution of bilingual experience may be a challenging task, because little is known about what experiences improve executive function (e.g., Owen et al., 2010; Valian, 2015), but investigating such experiences in greater depth may shed light on the complex relationship between bilingualism and cognitive ability.

Author note The authors thank David MacKinnon for his input on post-hoc power analyses. S.C.W. and S.D.G. were supported by NIH/ NICHD Grant R01 HD075800-03 to S.D.G.

\section{Appendix A}

Table 3 Main effects and interactions for Experiment 1

\begin{tabular}{|c|c|c|c|c|c|}
\hline & $F$ Value & $p$ Value & $\eta_{\mathrm{p}}^{2}$ & $1-\beta$ & $N$ Needed \\
\hline \multicolumn{6}{|l|}{ Overall Search } \\
\hline \multicolumn{6}{|l|}{ Black } \\
\hline Trial Type & 166.19 & $<.001$ & .856 & 1.000 & 6 \\
\hline Search Difficulty & 143.62 & $<.001$ & .837 & 1.000 & 6 \\
\hline Speaker Group & 0.26 & .612 & .009 & .068 & 1,064 \\
\hline Trial Type $\times$ Search Difficulty & 15.88 & $<.001$ & .362 & .947 & 21 \\
\hline Trial Type $\times$ Speaker Group & 0.02 & .893 & .001 & .052 & 9,629 \\
\hline Search Difficulty $\times$ Speaker Group & 0.96 & .388 & .033 & .126 & 286 \\
\hline Trial Type $\times$ Search Difficulty $\times$ Speaker Group & 0.31 & .735 & .011 & .073 & 870 \\
\hline \multicolumn{6}{|l|}{ Color } \\
\hline Trial Type & 94.93 & $<.001$ & .772 & 1.000 & 7 \\
\hline Search Difficulty & 278.40 & $<.001$ & .909 & 1.000 & 5 \\
\hline Speaker Group & 0.02 & .880 & .001 & .052 & 9,629 \\
\hline Trial Type $\times$ Search Difficulty & 72.16 & $<.001$ & .720 & 1.000 & 8 \\
\hline Trial Type $\times$ Speaker Group & 0.01 & .944 & .000 & $<.050$ & 96,341 \\
\hline Search Difficulty $\times$ Speaker Group & 0.25 & .778 & .009 & .068 & 1,064 \\
\hline Trial Type $\times$ Search Difficulty $\times$ Speaker Group & 0.25 & .778 & .009 & .068 & 1,064 \\
\hline \multicolumn{6}{|l|}{ First Fixations } \\
\hline \multicolumn{6}{|l|}{ Black } \\
\hline Search Difficulty & 74.26 & $<.001$ & .726 & 1.000 & 8 \\
\hline Speaker Group & 0.02 & .887 & .001 & .052 & 9,629 \\
\hline Search Difficulty $\times$ Speaker Group & 0.41 & .666 & .014 & .080 & 682 \\
\hline \multicolumn{6}{|l|}{ Color } \\
\hline Search Difficulty & 199.13 & $<.001$ & .877 & 1.000 & 5 \\
\hline Speaker Group & 0.56 & .459 & .020 & .094 & 476 \\
\hline Search Difficulty $\times$ Speaker Group & 1.02 & .368 & .035 & .131 & 269 \\
\hline \multicolumn{6}{|l|}{ Decision Times } \\
\hline \multicolumn{6}{|l|}{ Black } \\
\hline Search Difficulty & 8.01 & .001 & .222 & 697 & 37 \\
\hline Speaker Group & 0.27 & .606 & .010 & .071 & 957 \\
\hline Search Difficulty $\times$ Speaker Group & 3.06 & .055 & .098 & .315 & 92 \\
\hline \multicolumn{6}{|l|}{ Color } \\
\hline Search Difficulty & 47.18 & $<.001$ & .636 & 1.000 & 9 \\
\hline Speaker Group & 0.06 & .808 & .002 & .054 & 4,811 \\
\hline Search Difficulty $\times$ Speaker Group & 1.43 & .248 & .050 & .171 & 187 \\
\hline
\end{tabular}




\section{Appendix B}

Table 4 Main effects and interactions for Experiment 2

\begin{tabular}{|c|c|c|c|c|c|}
\hline & $F$ Value & $p$ Value & $\eta_{\mathrm{p}}^{2}$ & $1-\beta$ & $N$ Needed \\
\hline \multicolumn{6}{|l|}{ Overall Search } \\
\hline Condition & 5.13 & .008 & .128 & .378 & 87 \\
\hline Search Difficulty & 310.23 & $<.001$ & .899 & 1.000 & 6 \\
\hline Speaker Group & 0.03 & .857 & .001 & .052 & 11,929 \\
\hline Condition $\times$ Search Difficulty & 0.49 & .744 & .014 & .074 & 846 \\
\hline Condition $\times$ Speaker Group & 0.63 & .537 & .018 & .082 & 656 \\
\hline Search Difficulty $\times$ Speaker Group & 0.05 & .949 & .001 & .052 & 11,929 \\
\hline Condition $\times$ Search Difficulty $\times$ Speaker Group & 2.61 & .038 & .069 & .200 & 166 \\
\hline \multicolumn{6}{|l|}{ First Fixations } \\
\hline Condition & 1.37 & .261 & .038 & .123 & 307 \\
\hline Search Difficulty & 274.86 & $<.001$ & .887 & 1.000 & 6 \\
\hline Speaker Group & 0.88 & .353 & .025 & .095 & 471 \\
\hline Condition $\times$ Search Difficulty & 0.48 & .751 & .014 & .074 & 846 \\
\hline Condition $\times$ Speaker Group & 0.19 & .828 & .005 & .058 & 2,380 \\
\hline Search Difficulty $\times$ Speaker Group & 1.11 & .337 & .031 & .108 & 378 \\
\hline Condition $\times$ Search Difficulty $\times$ Speaker Group & 1.16 & .333 & .032 & .110 & 366 \\
\hline \multicolumn{6}{|l|}{ Decision Times } \\
\hline Condition & 16.38 & $<.001$ & .319 & .894 & 31 \\
\hline Search Difficulty & 91.35 & $<.001$ & .723 & 1.000 & 9 \\
\hline Speaker Group & 0.10 & .759 & .003 & .055 & 3,972 \\
\hline Condition $\times$ Search Difficulty & 0.84 & .500 & .024 & .093 & 491 \\
\hline Condition $\times$ Speaker Group & 1.34 & .267 & .037 & .121 & 316 \\
\hline Search Difficulty $\times$ Speaker Group & 0.03 & .967 & .001 & .052 & 11,929 \\
\hline Condition $\times$ Search Difficulty $\times$ Speaker Group & 2.28 & .064 & .061 & .179 & 189 \\
\hline
\end{tabular}

\section{Appendix C}

Table 5 Main effects and interactions for Experiment 3

\begin{tabular}{|c|c|c|c|c|c|}
\hline & $F$ Value & $p$ Value & $\eta_{\mathrm{p}}^{2}$ & $1-\beta$ & $N$ Needed \\
\hline \multicolumn{6}{|l|}{ Overall Search } \\
\hline Updating Condition & 21.58 & $<.001$ & .324 & .968 & 35 \\
\hline Search Difficulty & 28.15 & $<.001$ & .385 & .994 & 28 \\
\hline Speaker Group & 1.15 & .288 & .025 & .105 & 538 \\
\hline Condition $\times$ Search Difficulty & 1.59 & .149 & .034 & .129 & 394 \\
\hline Condition $\times$ Speaker Group & 2.87 & .039 & .060 & .210 & 220 \\
\hline Search Difficulty $\times$ Speaker Group & 3.31 & .041 & .069 & .241 & 191 \\
\hline Condition $\times$ Search Difficulty $\times$ Speaker Group & 1.70 & .120 & .036 & .135 & 372 \\
\hline \multicolumn{6}{|l|}{ First Fixations } \\
\hline Updating Condition & 13.80 & $<.001$ & .216 & .785 & 56 \\
\hline Search Difficulty & 42.15 & $<.001$ & .457 & .999 & 22 \\
\hline Speaker Group & 0.02 & .900 & .000 & $<.050$ & 136,236 \\
\hline Condition $\times$ Search Difficulty & 1.35 & .233 & .026 & .108 & 517 \\
\hline Condition $\times$ Speaker Group & 0.66 & .578 & .013 & .077 & 1,041 \\
\hline Search Difficulty $\times$ Speaker Group & 2.31 & .104 & .044 & .158 & 303 \\
\hline Condition $\times$ Search Difficulty $\times$ Speaker Group & 0.37 & .903 & .007 & .064 & 1,939 \\
\hline \multicolumn{6}{|l|}{ Decision Times } \\
\hline Updating Condition & 35.52 & $<.001$ & .404 & .997 & 26 \\
\hline Search Difficulty & 1.90 & .155 & .036 & .135 & 372 \\
\hline Speaker Group & 6.54 & .014 & .114 & .415 & 113 \\
\hline Condition $\times$ Search Difficulty & 1.36 & .230 & .025 & .105 & 538 \\
\hline Condition $\times$ Speaker Group & 1.75 & .158 & .033 & .126 & 406 \\
\hline Search Difficulty $\times$ Speaker Group & 1.59 & .208 & .030 & .118 & 447 \\
\hline Condition $\times$ Search Difficulty $\times$ Speaker Group & 2.67 & .015 & .050 & .177 & 266 \\
\hline
\end{tabular}




\section{Appendix D}

Table 6 Means, standard deviations, and confidence intervals for normative measures

\begin{tabular}{llr}
\hline & Raven's Advanced Progressive Matrices & Symmetry Span Task \\
\hline Experiment 1 & & \\
$\quad$ Bilingual & $9.27(2.69)[7.77,10.77]$ & $26.27(8.55)[22.46,30.08]$ \\
$\quad$ Monolingual & $8.27(2.99)[6.77,9.77]$ & $21.60(5.54)[17.79,25.41]$ \\
Experiment 2 & $7.83(3.29)[6.07,9.60]$ & $28.00(11.15)[22.95,33.05]$ \\
$\quad$ Bilingual & $9.42(4.03)[7.70,11.14]$ & $29.89(9.95)[24.98,34.81]$ \\
$\quad$ Monolingual & & \\
Experiment 3 & $8.31(3.57)[6.50,10.13]$ & $26.13(10.12)[20.93,31.32]$ \\
$\quad$ Bilingual & $7.36(3.59)[5.81,8.91]$ & $25.41(10.32)[20.98,29.84]$ \\
Monolingual & &
\end{tabular}

Standard deviations are in parentheses, $95 \%$ confidence intervals are shown in brackets.

\section{Appendix E}

Table 7 Means, standard deviations, and confidence intervals for Experiment 2

\begin{tabular}{|c|c|c|c|}
\hline & \multicolumn{3}{|l|}{ Visual Search Difficulty } \\
\hline & Easy & Medium & Difficult \\
\hline \multicolumn{4}{|l|}{ Overall Search } \\
\hline \multicolumn{4}{|l|}{ Color } \\
\hline Bilingual & $2,251.9(302.5)[2,093.3,2,410.6]$ & $3,798.2(726.3)[3,490.6,4,105.8]$ & $4,876.3(1,104.9)[4,412.6,5,339.9]$ \\
\hline Monolingual & $2,372.6(356.9)[2,218.1,2,526.9]$ & $3,926.1(552.4)[3,626.8,4,225.5]$ & $4,524.7(820.1)[4,073.4,4,975.9]$ \\
\hline \multicolumn{4}{|l|}{ Shape } \\
\hline Bilingual & $2,341.6(336.5)[2,165.1,2,518.1]$ & $3,991.5(916.8)[3,546.6,4,436.3]$ & $4,563.3(847.0)[4,182.6,4,944.1]$ \\
\hline Monolingual & $2,347.2(396.9)[2,175.5,2,518.9]$ & $3,907.9$ (941.8) [3,474.9, 4,340.9] & $4,640.5(744.0)[4,269.9,5,011.1]$ \\
\hline \multicolumn{4}{|l|}{ Switch } \\
\hline Bilingual & $2,502.9(381.6)[2,307.9,2,697.8]$ & $4,191.0(598.6)[3,877.2,4,504.8]$ & $4,596.9(845.5)[4,161.2,5,032.6]$ \\
\hline Monolingual & $2,413.9$ (430.4) [2,224.2, 2,603.7] & $4,160.4(705.5)[3,855.0,4,465.8]$ & $5,057.5(968.0)[4,633.4,5,481.6]$ \\
\hline \multicolumn{4}{|l|}{ First Fixations } \\
\hline \multicolumn{4}{|l|}{ Color } \\
\hline Bilingual & $1,541.3(467.7)[1,353.5,1,729.0]$ & $2,407.5$ (479.9) $[2,164.9,2,650.2]$ & $3,121.9$ (814.9) $[2,797.4,3,446.4]$ \\
\hline Monolingual & $1,534.0(304.5)[1,351.3,1,716.8]$ & $2,750.8(531.6)[2,514.6,2,986.9]$ & $3,107.9(516.9)[2,792.1,3,423.8]$ \\
\hline \multicolumn{4}{|l|}{ Shape } \\
\hline Bilingual & $1,548.4(269.4)[1,416.4,1,680.4]$ & $2,711.9$ (599.4) $[2,412.8,3,010.9]$ & $3,168.7(501.0)[2,895.1,3,442.3]$ \\
\hline Monolingual & $1,535.9(281.8)[1,407.4,1,664.4]$ & $2,796.6(648.3)[2,505.5,3,087.7]$ & $3,226.5(631.2)[2,960.2,3,492.8]$ \\
\hline \multicolumn{4}{|l|}{ Switch } \\
\hline Bilingual & $1,564.5(272.5)[1,439.1,1,690.0]$ & $2,694.5$ (410.2) [2,477.0, 2,911.9] & $3,026.0$ (490.7) $[2,744.6,3,307.5]$ \\
\hline Monolingual & $1,473.6(252.2)[1,351.4,1,595.7]$ & $2,772.5(492.5)[2,560.9,2,984.1]$ & $3,320.2(667.3)[3,046.3,3,594.2]$ \\
\hline \multicolumn{4}{|l|}{ Decision Times } \\
\hline \multicolumn{4}{|l|}{ Color } \\
\hline Bilingual & $850.2(187.9)[763.3,937.1]$ & $1,246.5(328.8)[1,079.9,1,413.2]$ & $1,669.0$ (451.9) $[1,439.4,1,898.6]$ \\
\hline Monolingual & 873.8 (175.4) [789.2, 958.4] & $1,226.1(365.8)[1,063.9,1,388.3]$ & $1,382.8(504.8)[1,159.3,1,606.3]$ \\
\hline \multicolumn{4}{|l|}{ Shape } \\
\hline Bilingual & $790.4(151.8)[700.8,879.9]$ & $1,117.1(452.5)[932.6,1,301.6]$ & $1,255.8(375.5)[1,038.6,1,473.1]$ \\
\hline Monolingual & $784.1(215.2)[696.9,871.2]$ & $1,080.6(309.2)[901.1,1,260.2]$ & $1,312.9(321.7)[1,101.4,1,524.4]$ \\
\hline \multicolumn{4}{|l|}{ Switch } \\
\hline Bilingual & 993.4 (154.9) [892.3, 1094.4] & $1,311.3(252.4)[1,172.5,1,450.1]$ & $1,474.3(357.2)[1,252.0,1,696.5]$ \\
\hline Monolingual & $929.8(253.2)[831.5,1028.2]$ & $1,319.5(321.7)[1,184.4,1,454.6]$ & $1,597.9(546.8)[1,381.5,1,814.2]$ \\
\hline
\end{tabular}

Standard deviations are in parentheses; $95 \%$ confidence intervals are shown in brackets. 


\section{Appendix F}

Table 8 Means, standard deviations, and confidence intervals for Experiment 3

\begin{tabular}{|c|c|c|c|}
\hline & \multicolumn{3}{|l|}{ Visual Search Difficulty } \\
\hline & Easy & Medium & Difficult \\
\hline \multicolumn{4}{|l|}{ Overall Search } \\
\hline \multicolumn{4}{|l|}{ Control } \\
\hline Bilingual & $2,686.6(558.6)[2,388.5,2,984.8]$ & $3,495.0(1,221.5)[3,066.3,3,923.7]$ & $3,644.7(1,088.6)[3,042.0,4,247.4]$ \\
\hline Monolingual & $3,015.6(865.7)[2,711.1,3,320.2]$ & $3,089.9$ (815.2) [2,651.9, 3,527.9] & $4,341.3(1,776.7)[3,725.7,4,956.9]$ \\
\hline \multicolumn{4}{|l|}{ Secondary Load } \\
\hline Bilingual & $2,833.0(816.6)[2,500.5,3,165.5]$ & $3,636.9(1,422.3)[3,164.0,4,129.8]$ & $4,019.4(1,017.2)[3,536.9,4,501.9]$ \\
\hline Monolingual & $3,122.4(800.3)[2,782.8,3,462.1]$ & $3,513.8(841.0)[3,020.5,4,007.1]$ & $3,732.7(1,317.4)[3,239.8,4,225.6]$ \\
\hline \multicolumn{4}{|l|}{ Updated Search } \\
\hline Bilingual & $4,098.9(1,969.6)[3,417.8,4,780.1]$ & $4,432.5(1,771.3)[3,837.6,5027.5]$ & $4,701.1(1,796.6)[4,081.1,5,321.1]$ \\
\hline Monolingual & $3,426.8(1,248.6)[2,731.0,4,122.6]$ & $3,585.0$ (100.9) [2,977.2, 4,192.8] & $4,150.1(1,130.2)[3,516.8,4,783.5]$ \\
\hline \multicolumn{4}{|l|}{ Dual Task } \\
\hline Bilingual & $4,522.8(1,852.5)[3,893.9,5,151.6]$ & $5,190.4(1,797.1)[4521.8,5859.0]$ & $4,694.3(1,289.7)[4,147.4,5,241.2]$ \\
\hline Monolingual & $3836.7(1,094.4)[3,194.4,4,479.1]$ & $4,350.6(1,425.8)[3,667.7,5033.6]$ & $4,827.9(1,371.3)[4,269.3,5,386.6]$ \\
\hline \multicolumn{4}{|l|}{ First Fixations } \\
\hline \multicolumn{4}{|l|}{ Control } \\
\hline Bilingual & $2,238.6(474.7)[2,021.3,2,456.0]$ & $2,575.8(633.1)[2,345.8,2805.8]$ & $2,820.4(737.5)[2,523.9,3,117.0]$ \\
\hline Monolingual & $2,365.0(669.7)[2,130.2,2,599.9]$ & $2,609.6(572.5)[2,361.1,2858.0]$ & $3,043.5$ (829.9) $[2,723.1,3,363.8]$ \\
\hline \multicolumn{4}{|l|}{ Secondary Load } \\
\hline Bilingual & $2,221.4(639.2)[1,976.1,2,466.6]$ & $2,786.0(811.4)[2,510.3,3,061.8]$ & $3,031.2(875.8)[2,699.9,3,362.5]$ \\
\hline Monolingual & $2,416.2(654.2)[2,151.3,2,681.1]$ & $2,631.4(611.9)[2,333.6,2929.3]$ & $3,009.8$ (869.1) $[2,652.0,3,367.6]$ \\
\hline \multicolumn{4}{|l|}{ Updated Search } \\
\hline Bilingual & $2,492.4(926.5)[2,126.4,2,858.3]$ & $2,878.7(835.1)[2,575.1,3,182.2]$ & $2,953.9$ (963.9) $[2,588.1,3,319.8]$ \\
\hline Monolingual & $2,673.9(1,006.6)[2,278.6,3,069.2]$ & $2,702.8(756.3)[2,374.9,3,030.7]$ & $3,140.9$ (963.6) $[2,745.7,3,536.0]$ \\
\hline \multicolumn{4}{|l|}{ Dual Task } \\
\hline Bilingual & $2,639.6(871.1)[2,314.9,2,964.4]$ & $3,462.2(1,121.4)[3,077.1,3,847.4]$ & $3,621.1(957.3)[3,254.6,3,987.7]$ \\
\hline Monolingual & $2,737.4(836.8)[2,386.7,3,088.2]$ & $3,144.9(873.1)[2,728.8,3,560.9]$ & $3,448.7$ (975.6) [3,052.8, 3,844.7] \\
\hline \multicolumn{4}{|l|}{ Decision Times } \\
\hline \multicolumn{4}{|l|}{ Control } \\
\hline Bilingual & $650.9(422.3)[513.4,788.2]$ & $765.3(508.1)[619.2,911.4]$ & $816.6(504.9)[647.6,985.6]$ \\
\hline Monolingual & $611.4(289.3)[460.5,762.4]$ & $562.0(162.2)[401.4,722.6]$ & $681.4(381.3)[495.6,867.1]$ \\
\hline \multicolumn{4}{|l|}{ Secondary Load } \\
\hline Bilingual & 699.5 (200.6) [618.4, 780.7] & $872.6(600.8)[694.8,1,050.5]$ & 856.6 (482.1) [698.0, 1,015.2] \\
\hline Monolingual & $605.1(237.0)[515.9,694.3]$ & $651.6(255.2)[456.2,847.1]$ & 644.9 (343.9) [470.5, 819.2] \\
\hline \multicolumn{4}{|l|}{ Updated Search } \\
\hline Bilingual & $1,330.6(1,090.3)[1,010.3,1,651.0]$ & $1,383.2(1,124.9)[1,045.5,1,720.9]$ & $1,626.5(1,009.9)[1,308.8,1,944.1]$ \\
\hline Monolingual & 824.8 (435.7) [472.7, 1176.9] & $886.7(528.4)[515.5,1,257.9]$ & $1,108.5(606.8)[759.3,1,457.7]$ \\
\hline \multicolumn{4}{|l|}{ Dual Task } \\
\hline Bilingual & $1,852.4(1,470.8)[1,430.2,2,274.5]$ & $1,666.0(1,185.7)[1,278.6,2,053.5]$ & $1,323.9(672.6)[1,043.8,2,274.5]$ \\
\hline Monolingual & $1,178.2(458.4)[714.2,1,642.3]$ & $1,224.1(826.9)[798.2,1,650.0]$ & $1,515.0(837.2)[1,207.1,1,822.9]$ \\
\hline
\end{tabular}

Standard deviations are in parentheses; $95 \%$ confidence intervals are shown in brackets. 


\section{Appendix G}

Table 9 Scaled Jeffrey-Zellner-Siow Bayes factors for speaker group main effects across experiments

\begin{tabular}{lll}
\hline & Scaled JZS Bayes Factor & Odds Favor \\
\hline Experiment 1 & & Null \\
$\quad$ Black & 3.41 & Null \\
$\quad$ Overall search response times & 3.78 & Null \\
$\quad$ First fixations & 3.41 & Null \\
$\quad$ Decision times & & Null \\
Color & 3.78 & Null \\
$\quad$ Overall search response times & 3.00 & Null \\
$\quad$ First fixations & 3.72 & Null \\
$\quad$ Decision times & 4.11 & Null \\
Experiment 2 & 2.84 & Null \\
$\quad$ Overall search response times & 3.98 & Null \\
$\quad$ First fixations & 3.90 & Alternate $(M+)$ \\
Decision times & 4.81 & \\
Experiment 3 & 3.42 & \\
$\quad$ Overall search response times & & \\
First fixations & & \\
Decision times & & \\
\hline
\end{tabular}

The scaling factor was set to 1 as a default. $M+=$ Monolingual advantage.

\section{Appendix H}

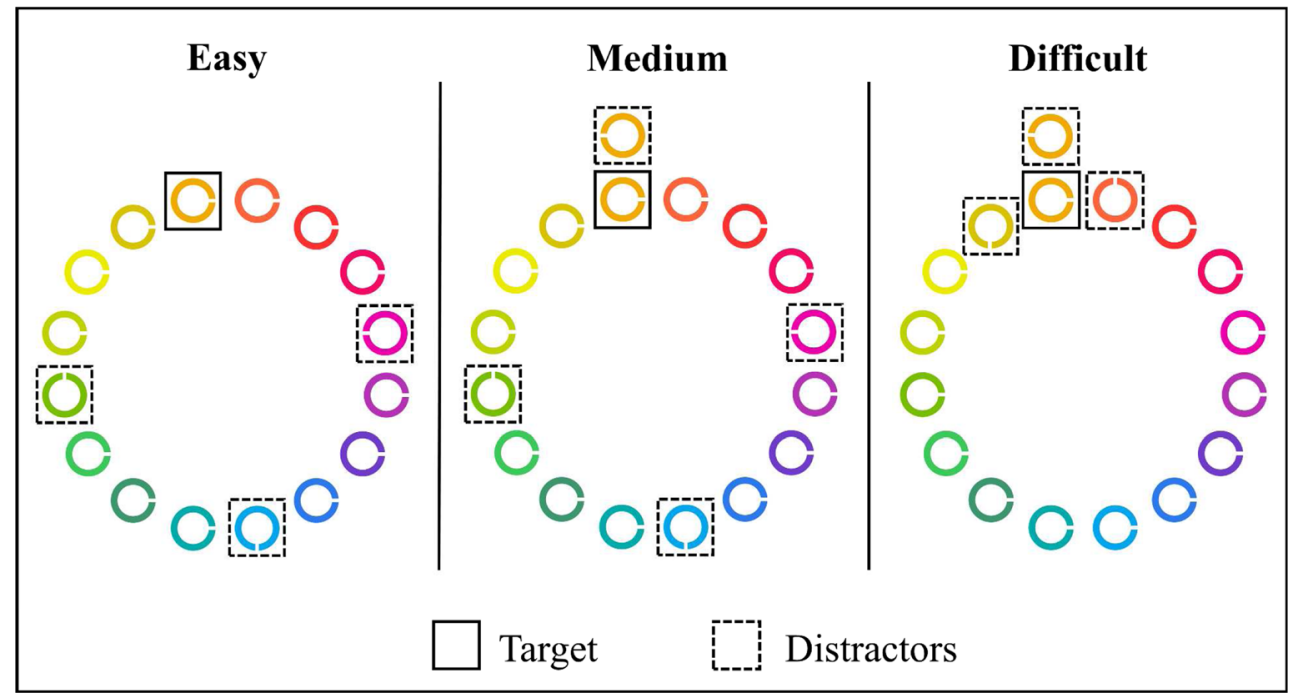

Fig. 7 Examples of stimulus selection in the easy, medium, and difficult conditions of Experiment 1 


\section{Appendix I}

A

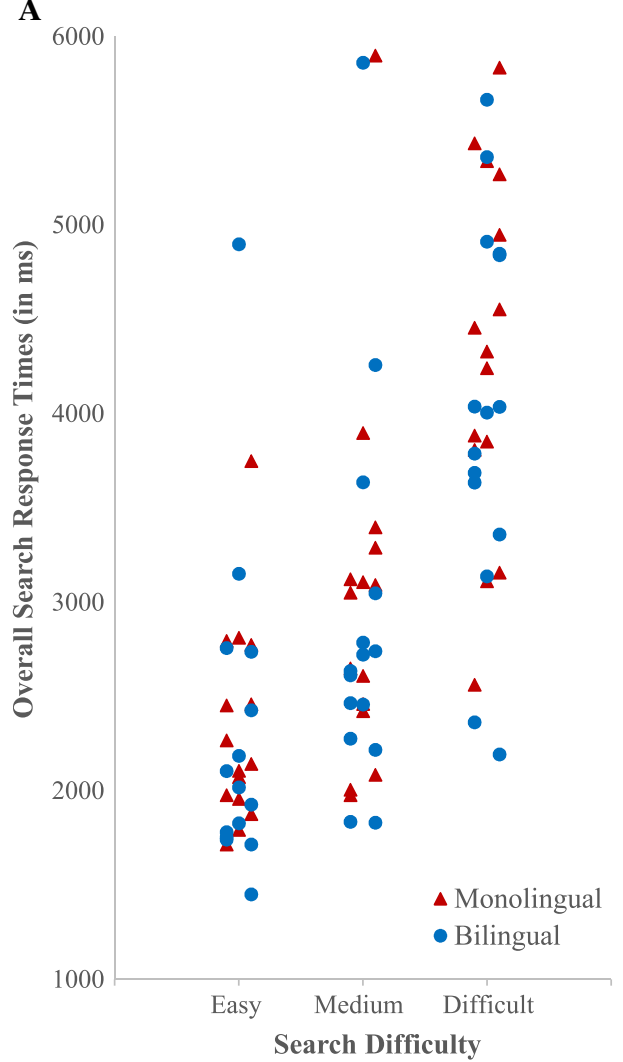

B

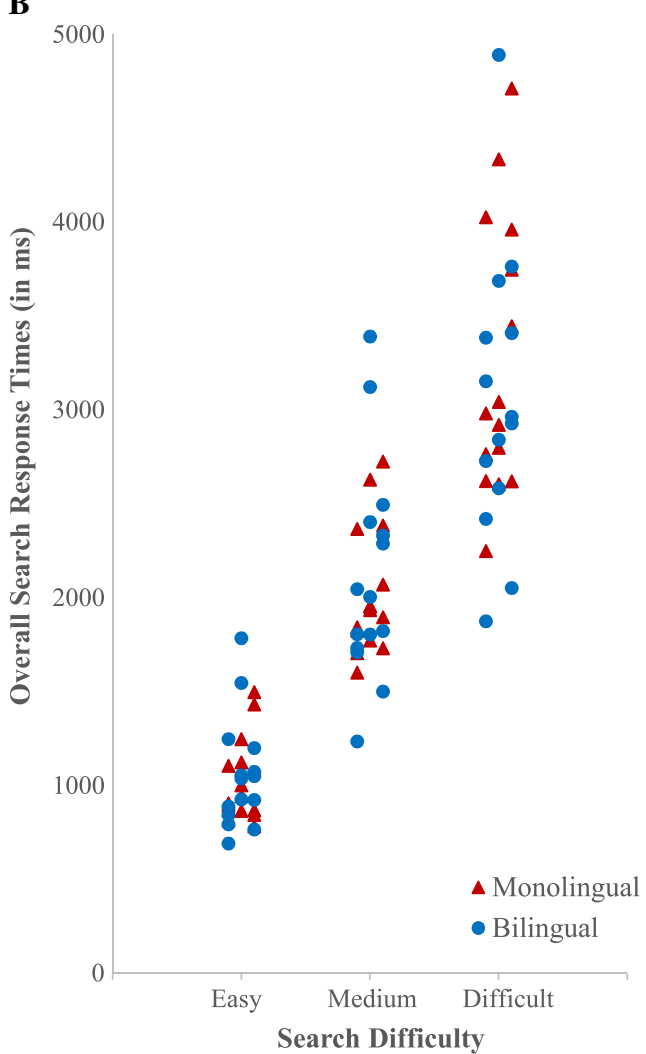

Fig. 8 Results from Experiment 1. Panel A shows individual search RTs in the Black Condition, panel B shows individual search RTs in the Color Condition 


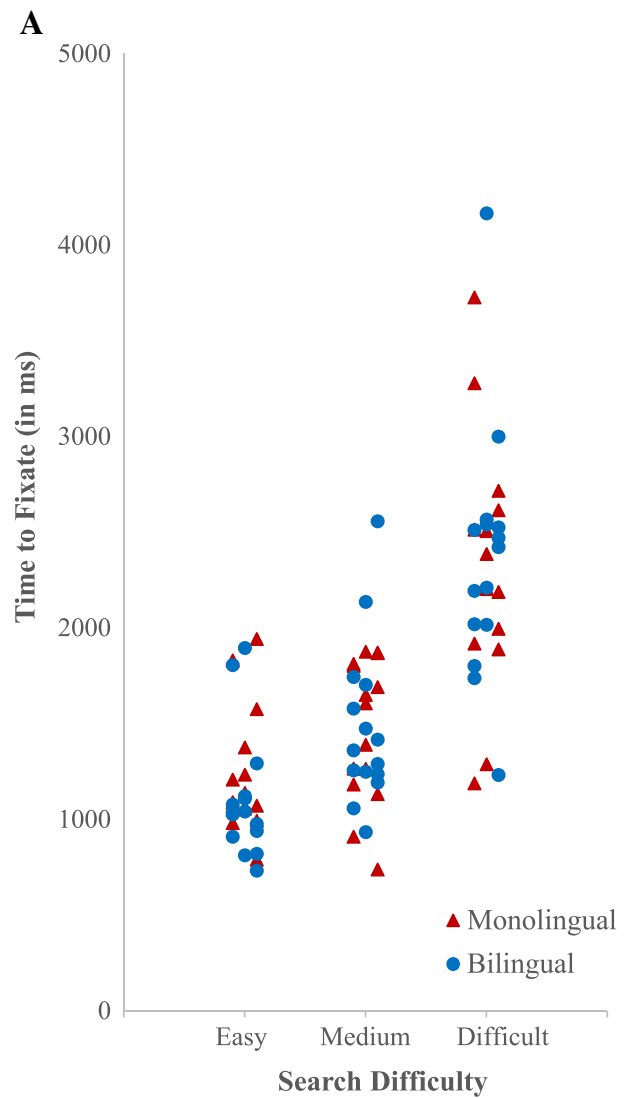

B

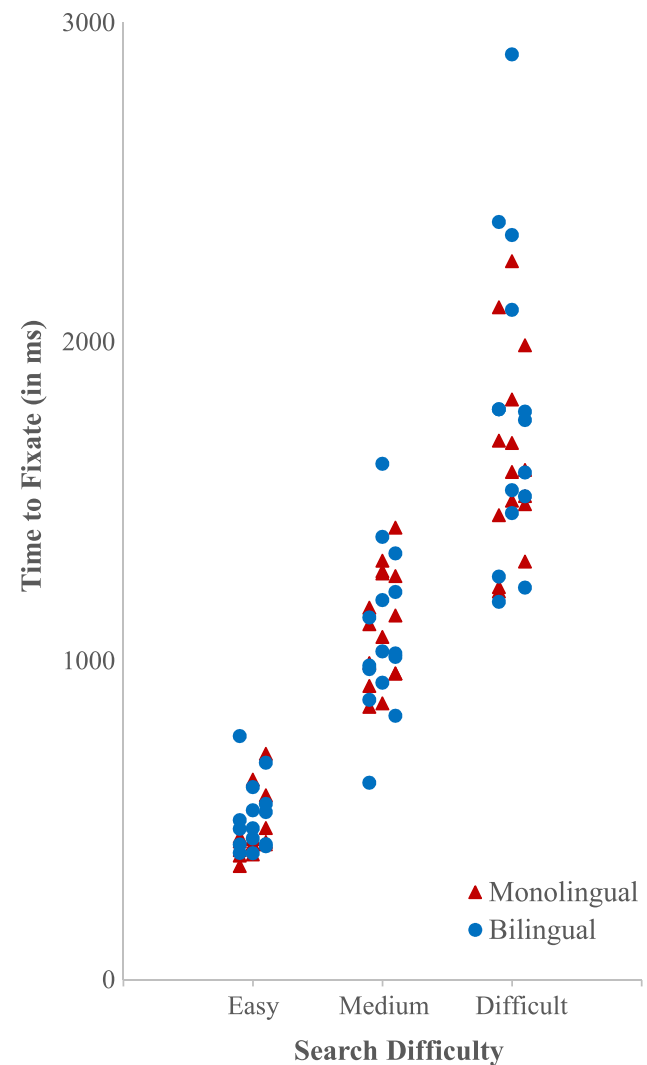

Fig. 9 Results from Experiment 1. Panel A shows individual first fixation times in the Black Condition, panel B shows individual first fixation times in the Color Condition 
A

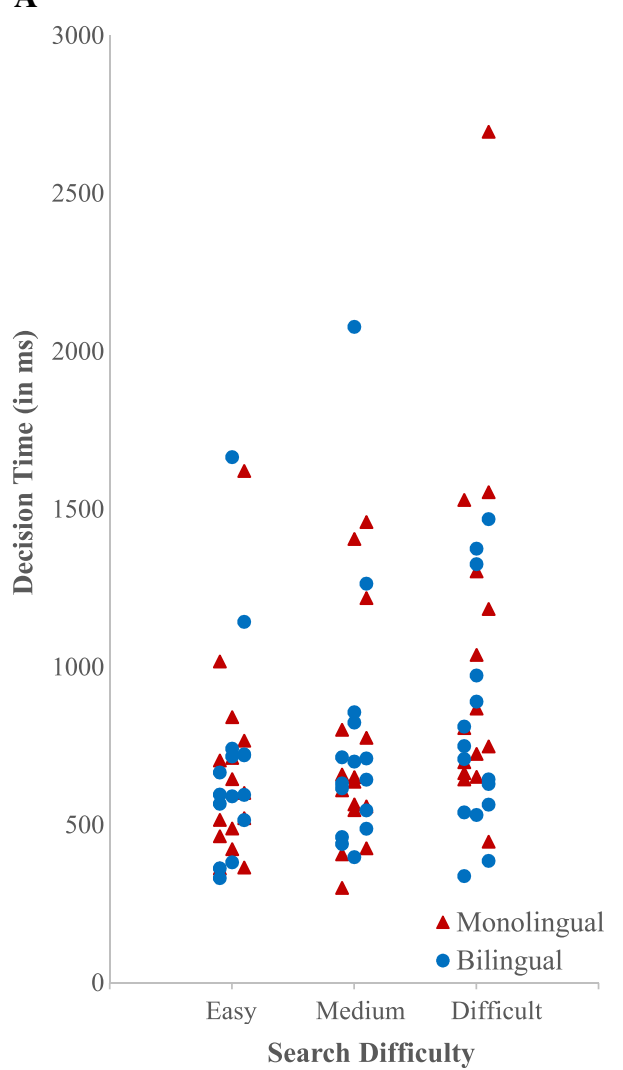

B

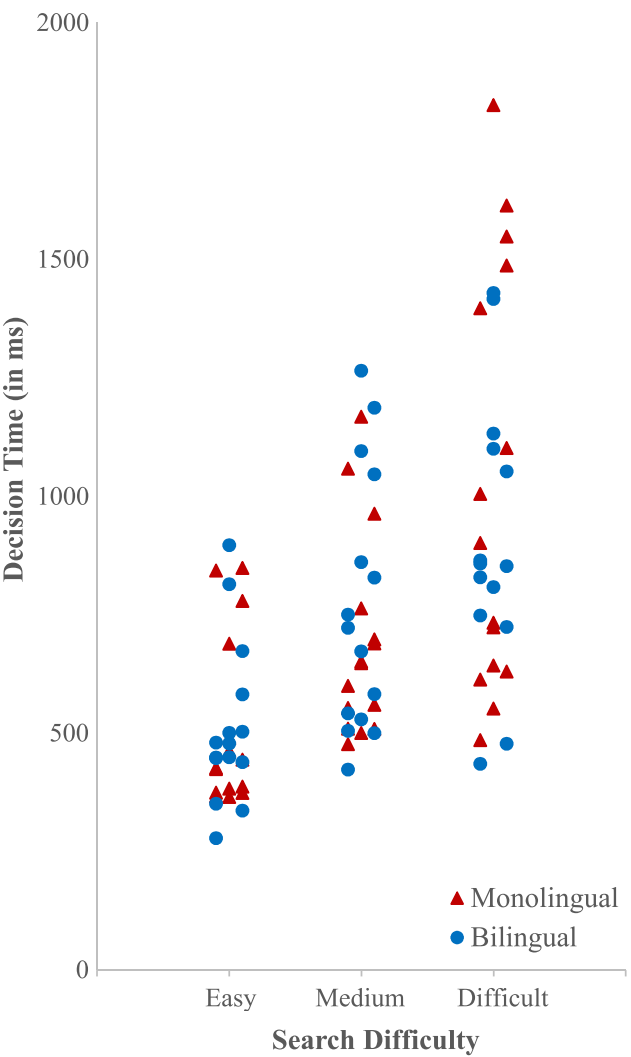

Fig. 10 Results from Experiment 1. Panel A shows individual decision times in the Black Condition, panel B shows individual decision times in the Color Condition

A

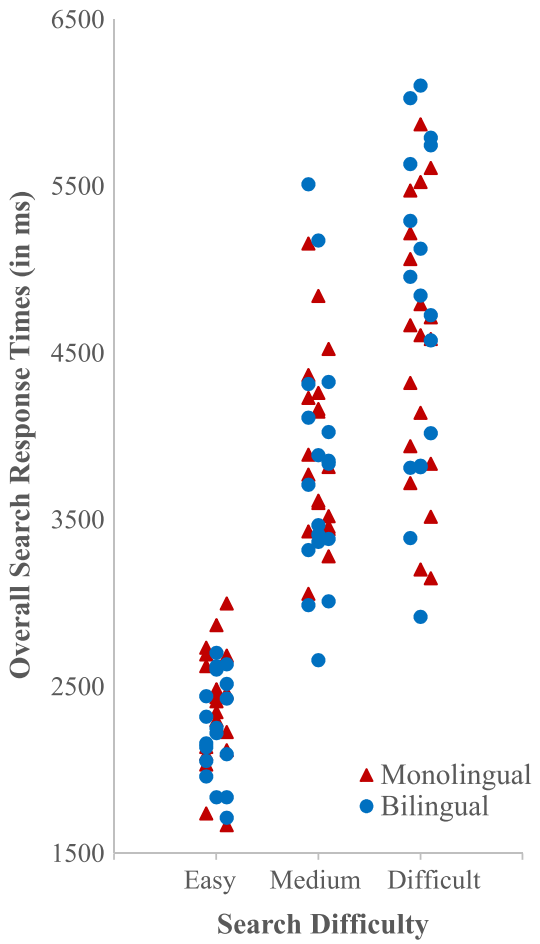

B

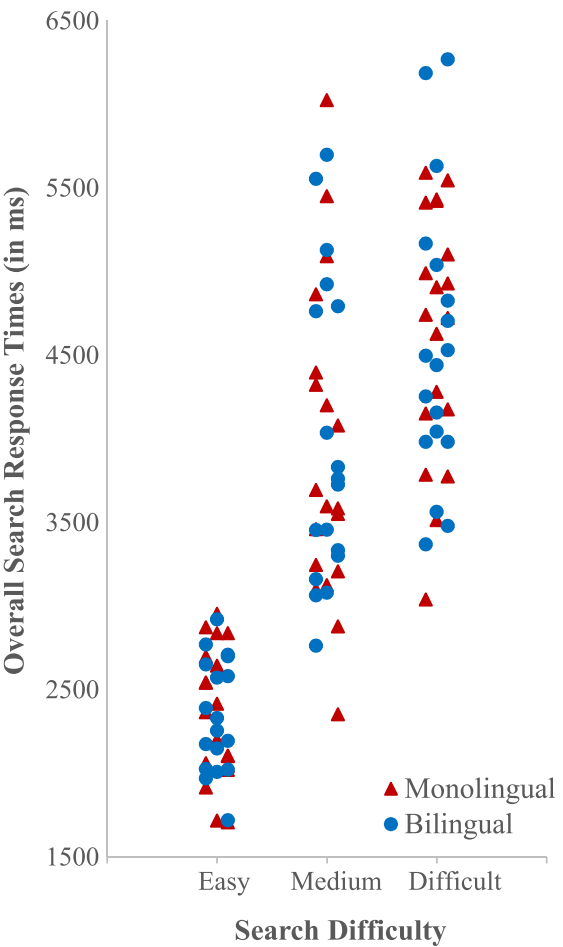

C

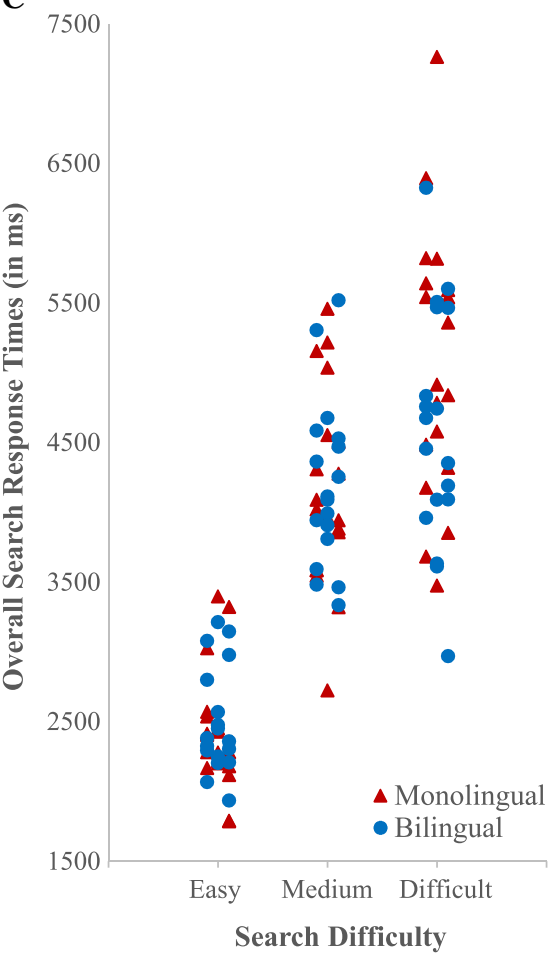

Fig. 11 Results from Experiment 2. Panel A shows individual search RTs in the Color Condition, panel B shows individual search RTs in the Shape Condition, panel C shows individual search RTs in the Switch Condition 

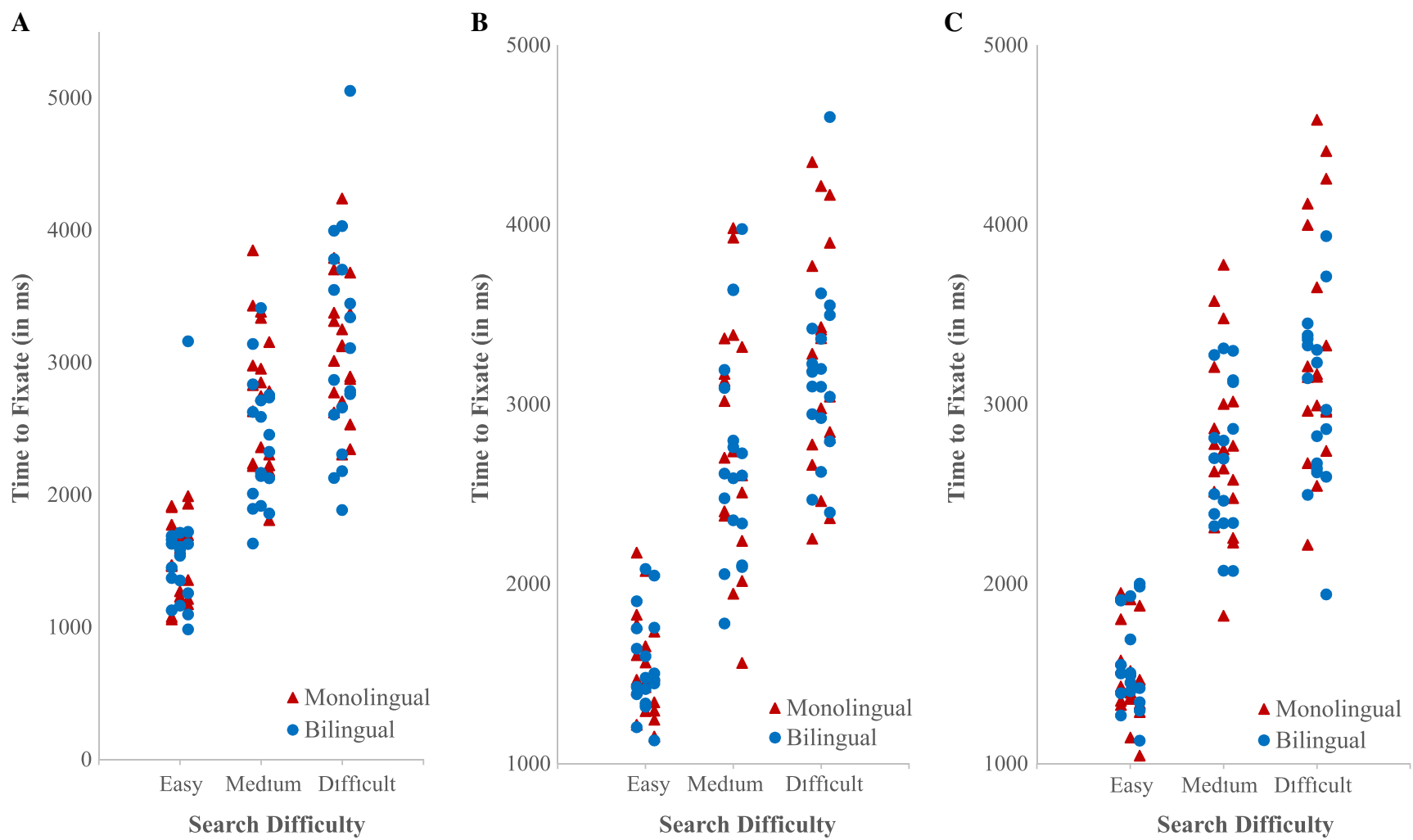

Fig. 12 Results from Experiment 2. Panel A shows individual first fixation times in the Color Condition, panel B shows individual first fixation times in the Shape Condition, panel C shows individual first fixation times in the Switch Condition
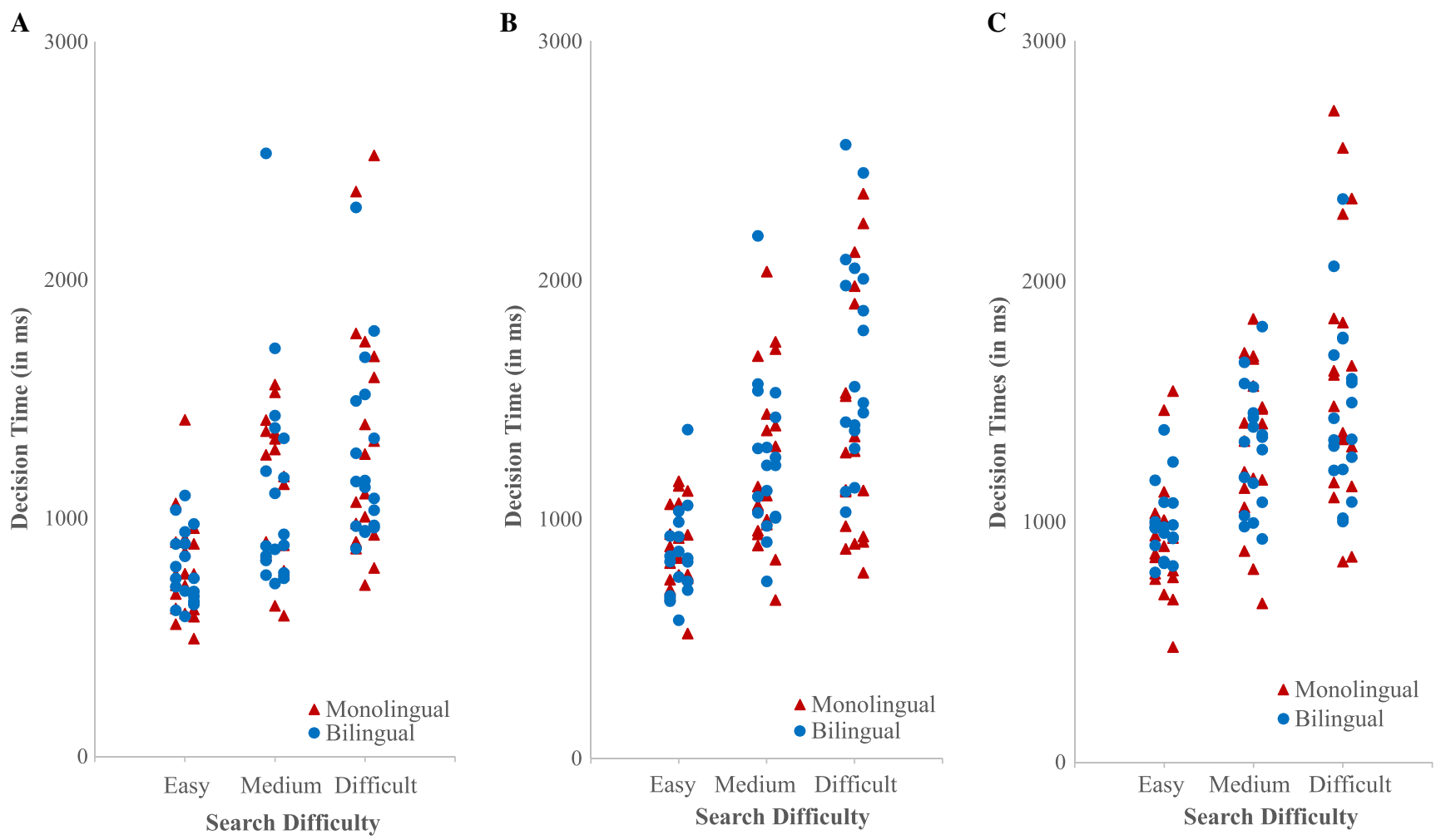

Fig. 13 Results from Experiment 2. Results from Experiment 2. Panel A shows individual decision times in the Color Condition, panel B shows individual first decision in the Shape Condition, panel C shows individual decision times in the Switch Condition 

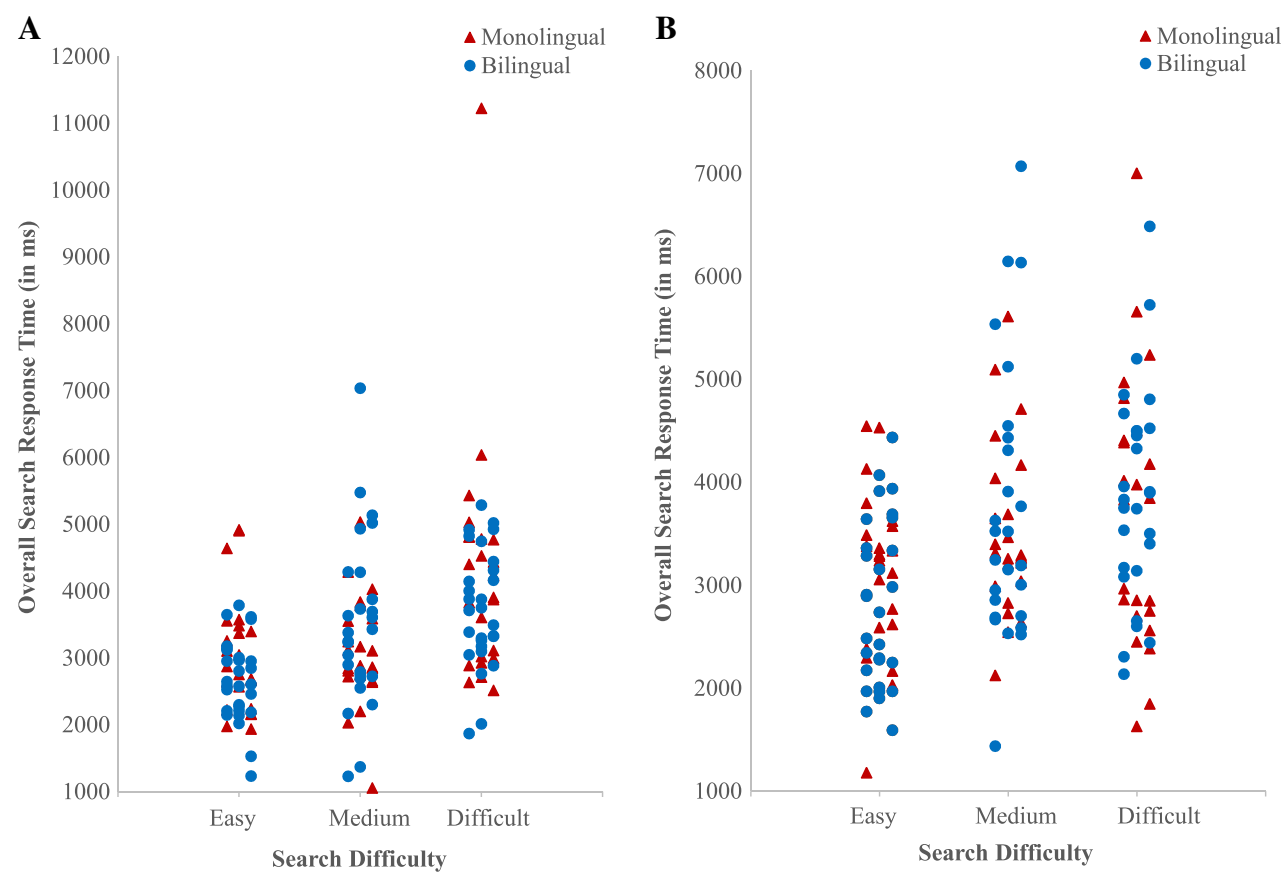

Fig. 14 Results from Experiment 3. Results from Experiment 3. Panel A shows individual search RTs in the Control Condition, panel B shows individual search RTs in the Secondary Load Condition
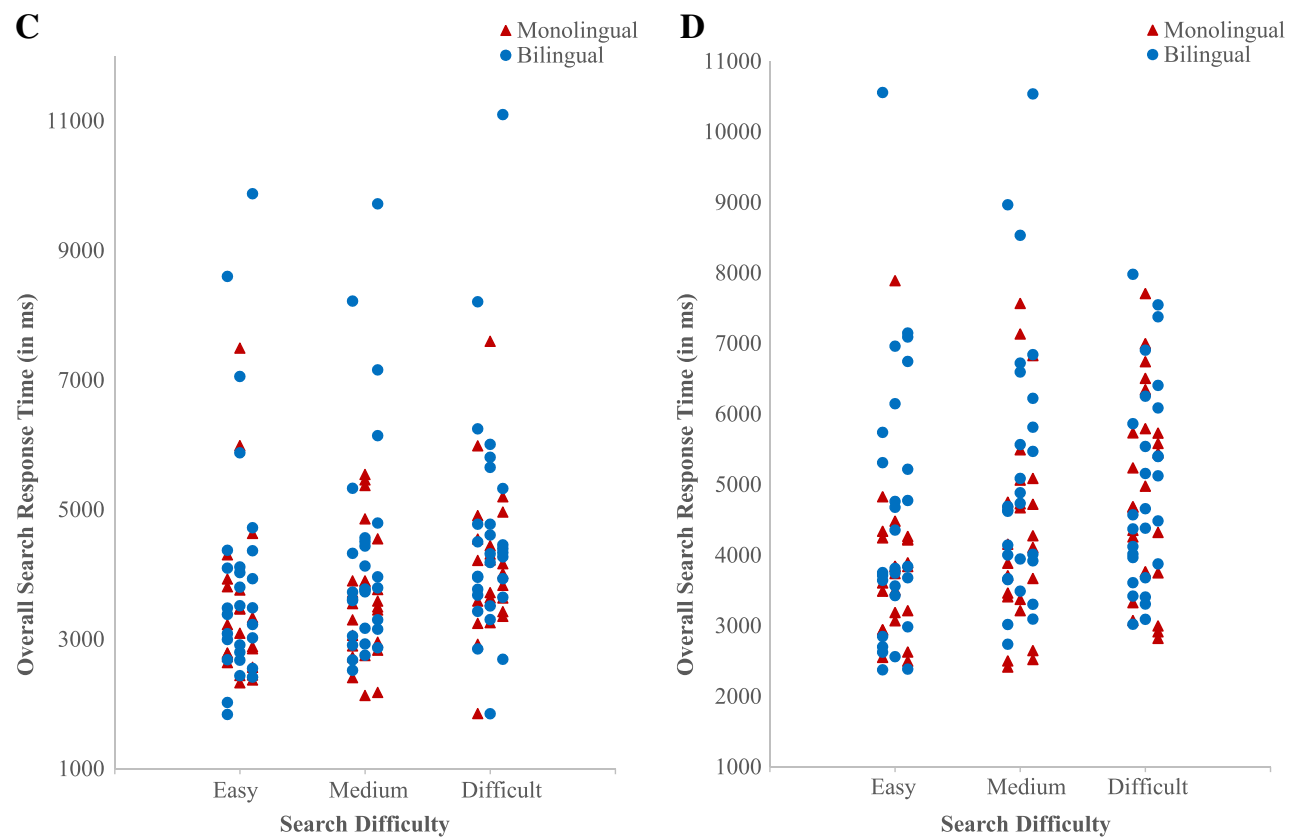

Fig. 15 Results from Experiment 3. Panel C shows individual search RTs in the Updated Search Condition, panel D shows individual search RTs in the Dual Task Condition 

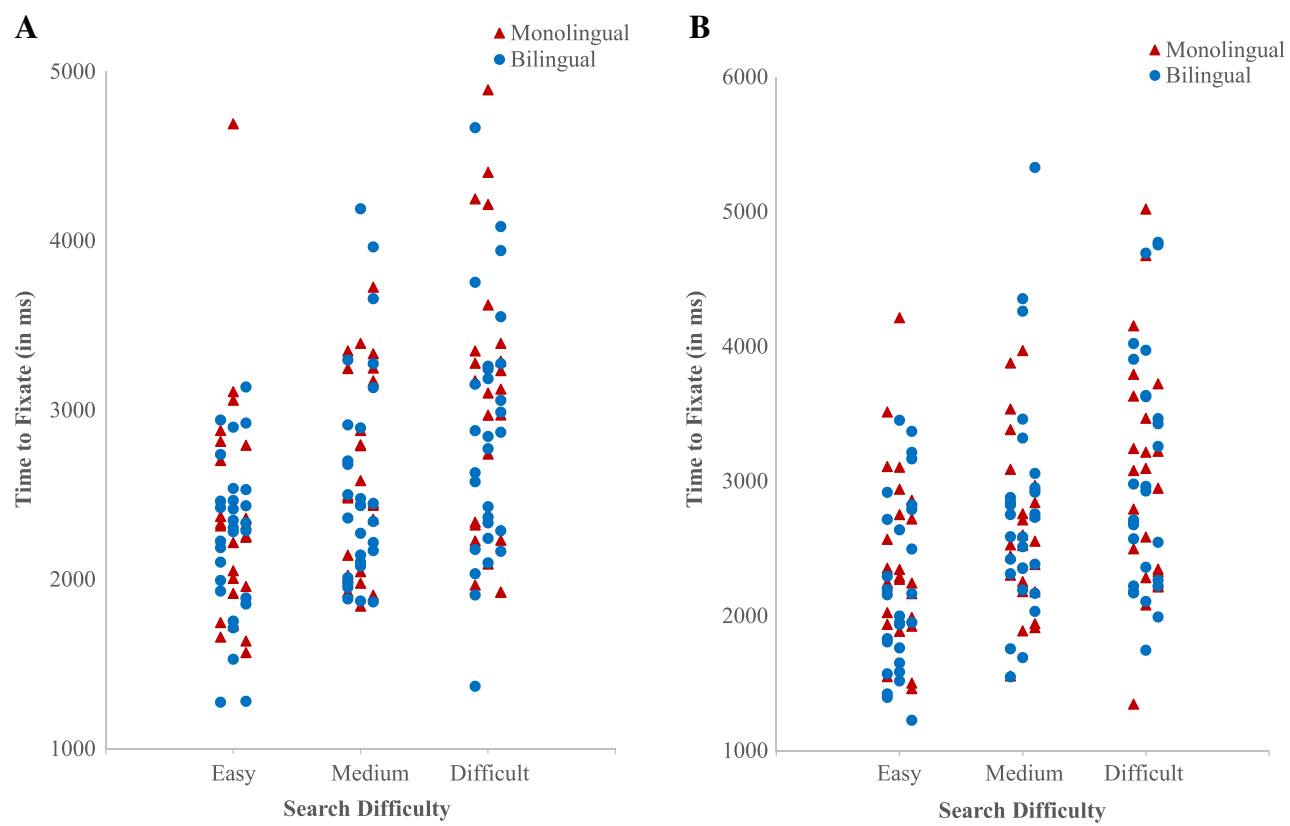

Fig. 16 Results from Experiment 3. Panel A shows individual first fixation times in the Control Condition, panel B shows individual first fixation times in the Secondary Load Condition
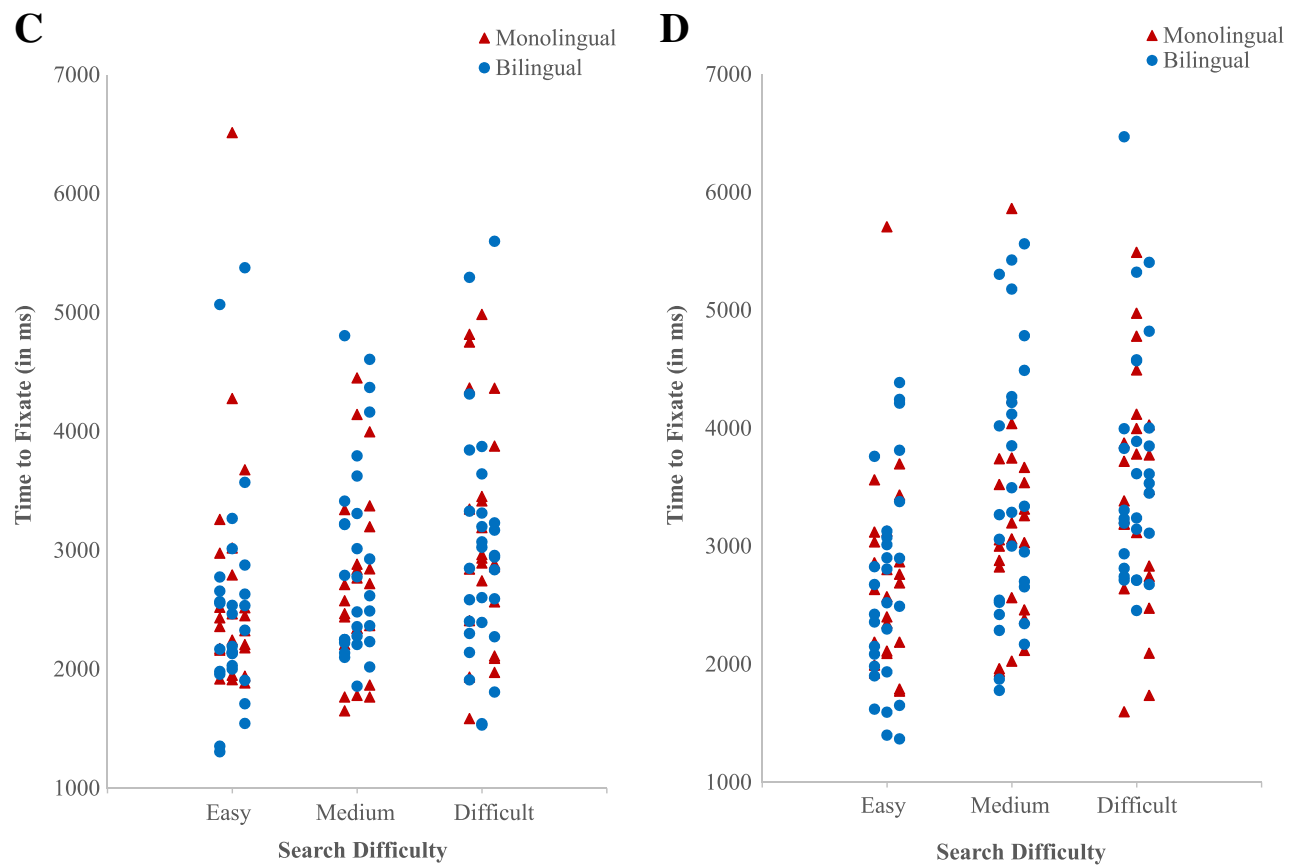

Fig. 17 Results from Experiment 3. Panel C shows individual first fixation times in the Updated Search Condition, panel D shows individual first fixation times in the Dual Task Condition 

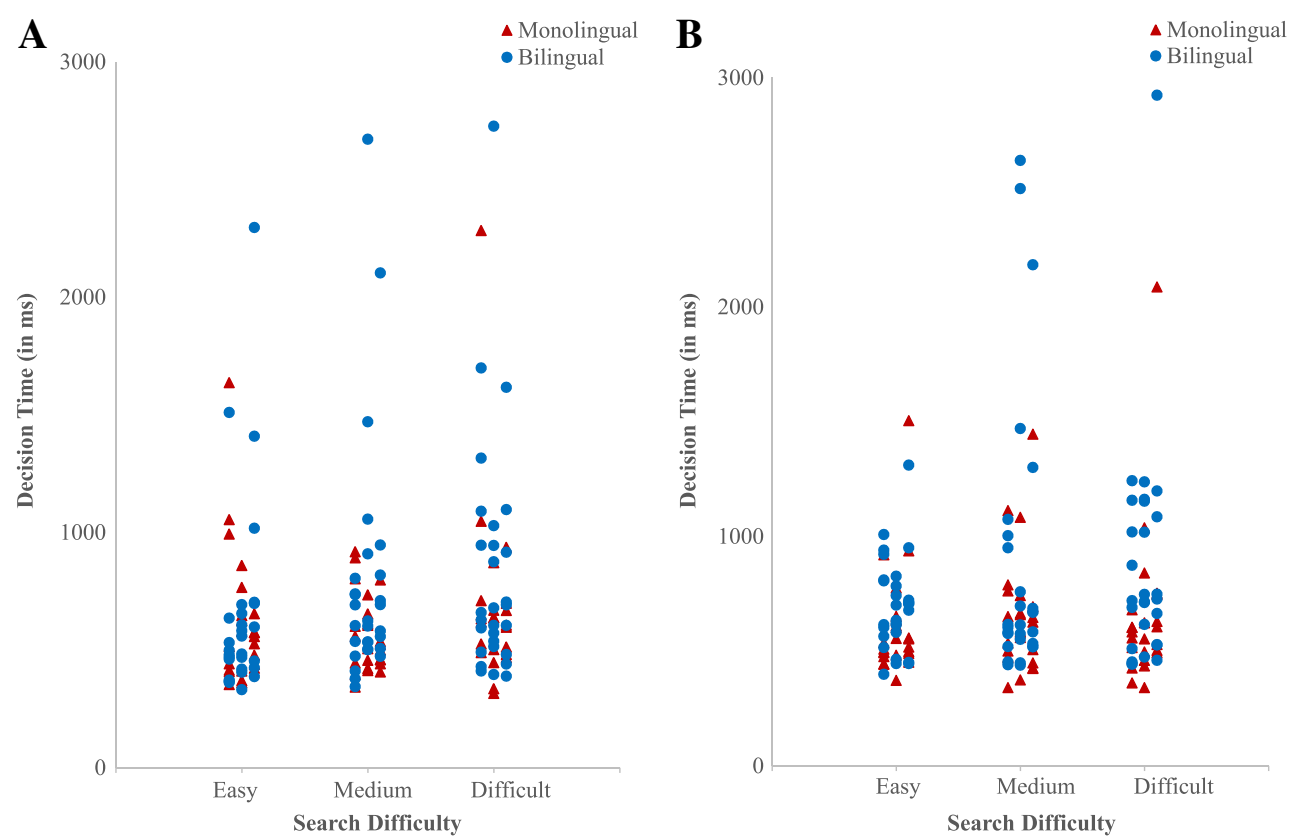

Fig. 18 Results from Experiment 3. Panel A shows individual decision times in the Control Condition, panel B shows individual decision times in the Secondary Load Condition
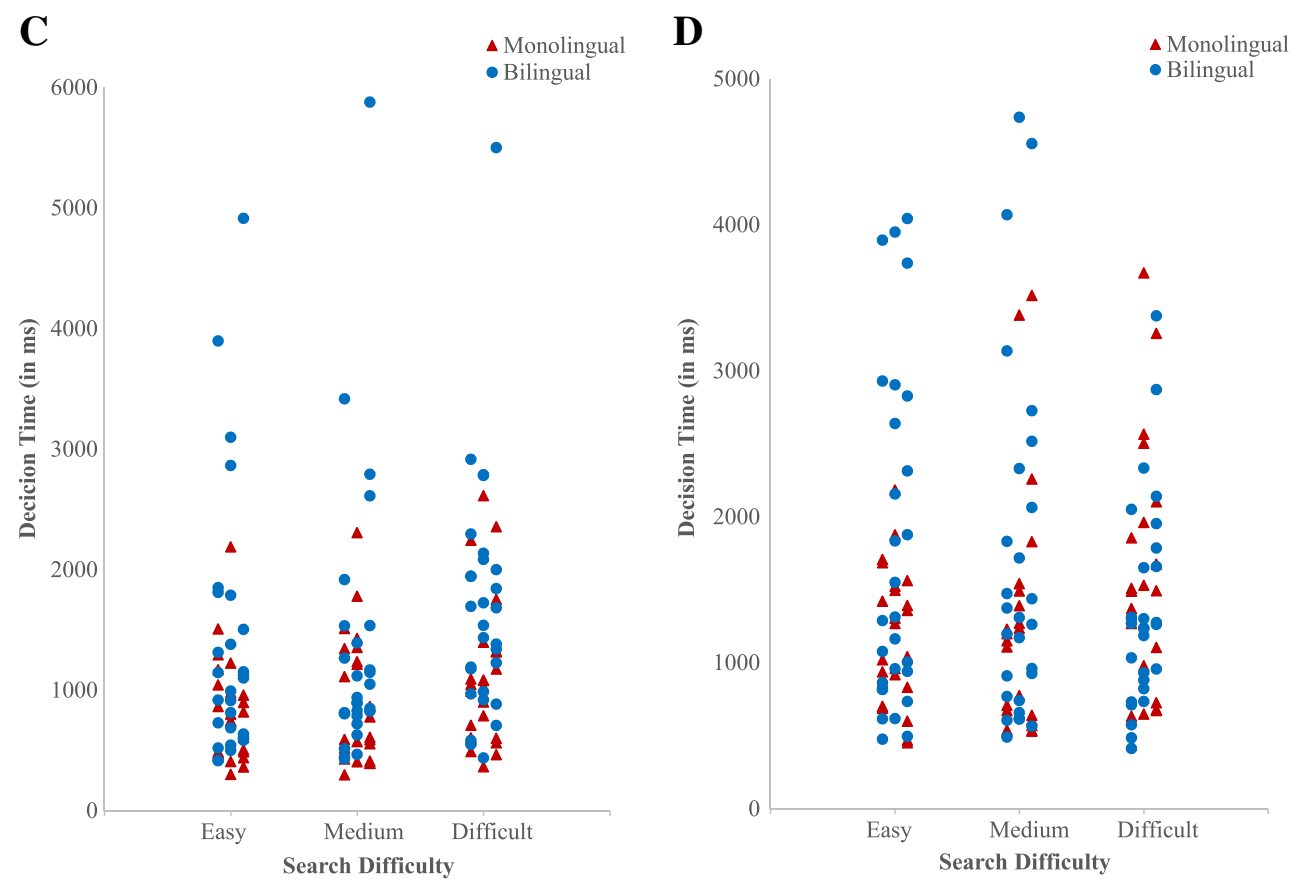

Fig. 19 Results from Experiment 3. Results from Experiment 3. Panel C shows individual decision times in the Updated Search Condition, panel D shows individual decision times in the Dual Task Condition 


\section{References}

Bialystok, E. (2006). Effect of bilingualism and computer video game experience on the Simon task. Canadian Journal of Experimental Psychology, 60, 68-79.

Bialystok, E. (2009). Bilingualism: The good, the bad, and the indifferent. Bilingualism: Language and Cognition, 12, 3-11. doi:10.1017/ S1366728908003477

Bialystok, E., Craik, F. I. M., \& Ruocco, A. C. (2006). Dual-modality monitoring in a classification task: The effects of bilingualism and aging. Quarterly Journal of Experimental Psychology, 59, 1968 1983. doi:10.1080/17470210500482955

Bialystok, E., Craik, F. I. M., \& Ryan, J. (2006). Executive control in a modified antisaccade task: Effects of aging and bilingualism. Journal of Experimental Psychology: Learning, Memory, and Cognition, 32, 1341-1354. doi:10.1037/0278-7393.32.6.1341

Bialystok, E., \& Viswanathan, M. (2009). Components of executive control with advantages for bilingual children in two cultures. Cognition, 112, 494-500. doi:10.1016/j.cognition.2009.06.014

Chabal, S., Schroeder, S. R., \& Marian, V. (2015). Audio-visual object search is changed by bilingual experience. Atteniton, Perception, \& Psychophysics, 77, 2684-2693. doi:10.3758/s13414-015-0973-7

Costa, A., Hernández, M., Costa-Faidella, J., \& Sebastián-Gallés, N. (2009). On the bilingual advantage in conflict processing: Now you see it, now you don't. Cognition, 113, 135-149. doi:10.1016/ j.cognition.2009.08.001

De Groot, A. M. B., Delmaar, P., \& Lupker, S. J. (2000). The processing of interlexical homographs in translation recognition and lexical decision: Support for non-selective access to bilingual memory. Quarterly Journal of Experimental Psychology, 53A, 397-428. doi:10.1080/713755891

Dijkstra, T., Van Jaarsveld, H., \& Ten Brinke, S. (1998). Interlingual homograph recognition: Effects of task demands and language intermixing. Bilingualism: Language and Cognition, 1, 51-66. doi:10.1017/S1366728998000121

Duncan, J., \& Humphreys, G. W. (1989). Visual search and stimulus similarity. Psychological Review, 96, 433-458. doi:10.1037/0033295X.96.3.433

Emmorey, K., Luk, G., Pyers, J. E., \& Bialystok, E. (2008). The source of enhanced cognitive control in bilinguals: Evidence from bimodal bilinguals. Psychological Science, 19, 1201-1206. doi:10.1111/j. 1467-9280.2008.02224.x

Friedman, N. P., Miyake, A., Corley, R. P., Young, S. E., Defries, J. C., \& Hewitt, J. K. (2006). Not all executive functions are related to intelligence. Psychological Science, 17, 172-179. doi:10.1111/j.14679280.2006.01681.x

Friesen, D. C., Latman, V., Calvo, A., \& Bialystok, E. (2015). Attention during visual search: The benefit of bilingualism. International Journal of Bilingualism, 19, 693-702. doi:10.1177/ 1367006914534331

Godwin, H. J., Walenchok, S., Houpt, J. W., Hout, M. C., \& Goldinger, S. D. (2015). Faster than the speed of rejection: Object identification processes during visual search for multiple targets. Journal of Experimental Psychology: Human Perception and Performance, 41, 1007-1020. doi:10.1037/xhp0000036

Gollan, T. H., Sandoval, T., \& Salmon, D. P. (2011). Cross-language intrusion errors in aging bilinguals reveal the link between executive control and language selection. Psychological Science, 22, 11551164. doi: $10.1177 / 095679761141700$

Green, D. W., \& Abutalebi, J. (2013). Language control in bilinguals: The adaptive control hypothesis. Journal of Cognitive Psychology, 25, 515-530. doi:10.1080/20445911.2013.796377

Hernández, M., Costa, A., \& Humphreys, G. W. (2012). Escaping capture: Bilingualism modulates distraction from working memory. Cognition, 122, 37-50. doi:10.1016/j.cognition.2011.08.002
Hilchey, M. D., \& Klein, R. M. (2011). Are there bilingual advantages on nonlinguistic interference tasks? Implications for the plasticity of executive control processes. Psychonomic Bulletin \& Review, 18, 625-658. doi:10.3758/s13423-011-0116-7

Hout, M. C., \& Goldinger, S. D. (2015). Target templates: The precision of mental representations affects attentional guidance and decisionmaking in visual search. Attention, Perception \& Psychophysics, 77, 128-149. doi:10.3758/s13414-014-07646

Hout, M. C., Walenchok, S. C., Goldinger, S. D., \& Wolfe, J. M. (2015). Failures of perception in the low-prevalence effect: Evidence from active and passive visual search. Journal of Experimental Psychology: Human Perception and Performance, 41, 977-994. doi:10.1037/xhp0000053

Ishihara, S. (1917). Tests for color blindness. Tokyo: Hongo Harukicho.

Kane, M. J., \& Engle, R. W. (2002). The role of prefrontal cortex in working-memory capacity, executive attention, and general fluid intelligence: An individual-differences perspective. Psychonomic Bulletin \& Review, 9, 637-671. doi:10.3758/BF03196323

Kane, M. J., Poole, B. J., Tuholski, S. W., \& Engle, R. W. (2006). Working memory capacity and the top-down control of visual search: Exploring the boundaries of "executive attention.". Journal of Experimental Psychology: Learning, Memory, and Cognition, 32, 749-777. doi:10.1037/0278-7393.32.4.749

Kousaie, S., \& Phillips, N. A. (2012). Ageing and bilingualism: Absence of a "bilingual advantage" in Stroop interference in a nonimmigrant sample. Quarterly Journal of Experimental Psychology, 65, 356369. doi:10.1080/17470218.2011.604788

Krantz, J. H. (2007). Dual Tasking Experiment (Version 1.1) [Software]. Retrieved August 6, 2009, from http://psych.hanover.edu/JavaTest/ CLE/Cognition/Cognition/dualtask_instructions.html

Kroll, J. F., Bobb, S. C., Misra, M., \& Guo, T. (2008). Language selection in bilingual speech: Evidence for inhibitory processes. Acta Psychologica, 128, 416-430. doi:10.1016/j.actpsy.2008.02.001

Macnamara, B. N., \& Conway, A. R. A. (2014). Novel evidence in support of the bilingual advantage: Influences of task demands and experience on cognitive control and working memory. Psychonomic Bulletin \& Review, 21, 520-525. doi:10.3758/ s13423-013-0524-y

Marian, V., Blumenfeld, H. K., \& Kaushanskaya, M. (2007). The language experience and proficiency questionnaire (LEAP-Q): Assessing language profiles in bilinguals and multilinguals. Journal of Speech Language and Hearing Research, 50, 940-968. doi:10.1044/1092-4388(2007/067)

Miyake, A., Friedman, N. P., Emerson, M. J., Witzki, A. H., Howerter, A., \& Wager, T. D. (2000). The unity and diversity of executive functions and their contributions to complex "frontal lobe" tasks: A latent variable analysis. Cognitive Psychology, 41, 49-100. doi:10. 1006/cogp.1999.0734

Moradzadeh, L., Blumenthal, G., \& Wiseheart, M. (2015). Musical training, bilingualism, and executive function: A closer look at task switching and dual-task performance. Cognitive Science, 39, 9921020. doi:10.1111/cogs. 12183

Owen, A. M., Hampshire, A., Grahn, J. A., Stenton, R., Dajani, S., Burns, A. S., \& Ballard, C. G. (2010). Putting brain training to the test. Nature, 465, 775-778. doi:10.1038/nature09042

Paap, K. R., \& Greenberg, Z. I. (2013). There is no coherent evidence for a bilingual advantage in executive processing. Cognitive Psychology, 66, 232-58. doi:10.1016/j.cogpsych.2012.12.002

Paap, K. R., Johnson, H. A., \& Sawi, O. (2015). Bilingual advantages in executive functioning either do not exist or are restricted to very specific and undetermined circumstances. Cortex, 69, 265-278. doi:10.1016/j.cortex.2015.04.014

Poole, B. J., \& Kane, M. J. (2009). Working-memory capacity predicts the executive control of visual search among distractors: The influences of sustained and selective attention. Quarterly Journal of 
Experimental Psychology, 62, 1430-1454. doi:10.1080/ 17470210802479329

Prior, A., \& Gollan, T. H. (2011). Good language-switchers are good taskswitchers: Evidence from Spanish-English and Mandarin-English bilinguals. Journal of the International Neuropsychological Society, 17, 682-691. doi:10.1017/S1355617711000580

Prior, A., \& MacWhinney, B. (2010). A bilingual advantage in task switching. Bilingualism: Language and Cognition, 13, 253-262. doi:10.1017/S1366728909990526

Ratiu, I., \& Azuma, T. (2015). Working memory capacity: Is there a bilingual advantage? Journal of Cognitive Psychology, 27, 1-11. doi:10.1080/20445911.2014.976226

Raven, J. C., Raven, J. E., \& Court, J. H. (1998). Progressive matrices. Oxford: Oxford Psychologists Press.

Rouder, J. N., Speckman, P. L., Sun, D., Morey, R. D., \& Iverson, G. (2009). Bayesian $t$ tests for accepting and rejecting the null hypothesis. Psychonomic Bulletin \& Review, 16, 225-237. doi:10.3758/ PBR.16.2.225

Schneider, W., Eschman, A., \& Zuccolotto, A. (2012). E-Prime user's guide. Pittsburgh: Psychology Software Tools.

Shipstead, Z., Lindsey, D. R. B., Marshall, R. L., \& Engle, R. W. (2014). The mechanisms of working memory capacity: Primary memory, secondary memory, and attention control. Journal of Memory and Language, 72, 116-141.

Singh, N., \& Mishra, R. K. (2012). Does language proficiency modulate oculomotor control? Evidence from Hindi-English bilinguals. Bilingualism: Language and Cognition, 15, 771-781. doi:10. 1017/S1366728912000065
Singh, N., \& Mishra, R. K. (2013). Second language proficiency modulates conflict-monitoring in an oculomotor Stroop task: Evidence from Hindi-English bilinguals. Frontiers in Psychology, 4(322), 1-10. doi:10.3389/fpsyg.2013.00322

Sobel, K. V., Gerrie, M. P., Poole, B. J., \& Kane, M. J. (2007). Individual differences in working memory capacity and visual search: The roles of top-down and bottom-up processing. Psychonomic Bulletin \& Review, 14, 840-845. doi:10.3758/BF03194109

Soveri, A., Rodriguez-Fornells, A., \& Laine, M. (2011). Is there a relationship between language switching and executive functions in bilingualism? Introducing a within group analysis approach. Frontiers in Psychology, 2(183), 1-8. doi:10.3389/fpsyg.2011. 00183

Stroud, M. J., Menneer, T., Cave, K. R., \& Donnelly, N. (2012). Using the dual-target cost to explore the nature of search target representations. Journal of Experimental Psychology: Human Perception and Performance, 38, 113-122. doi:10.1037/a0025887

Tat, M. J., \& Azuma, T. (2016). The effect of cognitive loads on hemispheric asymmetries in true and false memory. Laterality, 21, 50-75. doi:10.1080/1357650X.2015.1073297

Treisman, A. M., \& Gelade, G. (1980). A feature-integration theory of attention. Cognitive Psychology, 12, 97-136. doi:10.1016/00100285(80)90005-5

Unsworth, N., Heitz, R. P., Schrock, J. C., \& Engle, R. W. (2005). An automated version of the operation span task. Behavior Research Methods, 37, 498-505. doi:10.3758/BF03192720

Valian, V. (2015). Bilingualism and cognition. Bilingualism: Language and Cognition, 18, 3-24. doi:10.1017/S1366728914000522 